\title{
Scleractinian corals collected during 1998 from the Dampier Archipelago, Western Australia
}

\author{
Jane K. Griffith \\ Department of Aquatic Zoology (Marine Invertebrates), Western Australian Museum, \\ Francis Street, Perth, Western Australia 6000, Australia \\ email: c/o jane.fromont@museum.wa.gov.au
}

\begin{abstract}
During this study, 14 new records of zooxanthellate scleractinian coral species were recorded from the Dampier Archipelago, Western Australia. This brings the total number of these corals for the Dampier Archipelago to 229 species from 57 genera. The Dampier Archipelago is placed second only to Ashmore Reef in terms of number of coral species for a Western Australian reef. High species richness did not necessarily mean high coral cover. The first reported case of a hard coral containing mature ova during spring on the west coast of Australia is described. A complete updated list of azooxanthellate scleractinian corals recorded from the Dampier Archipelago is also presented ( 25 taxa in 6 families).
\end{abstract}

\section{INTRODUCTION}

Scientists from the Western Australian Museum first conducted surveys of the fauna of the Dampier Archipelago, including hard corals, during 19721974 and again during 1978 (Marsh, 1978). These surveys were concentrated around the Burrup Peninsula and the outer westerly islands such as Kendrew and Rosemary and only extended to $9 \mathrm{~m}$ in depth. Simpson (1988) recorded additional hard coral species from the archipelago during ecological fieldwork conducted by the Environmental Protection Authority in 1982-1985. He also surveyed around Kendrew and Rosemary Islands, as well as Hamersley Shoal and outer Legendre Island. From these earlier field trips and recent Western Australian Museum records, the corrected number of zooxanthellate scleractinian coral species recorded before this study was 215 from 56 genera (Marsh, 1978; Simpson, 1988; Veron, 1993; Veron and Marsh, 1988). The corrections to these published lists were made with reference to the revision of the Fungiidae by Hoeksema (1989).

The aim of this study was to conduct a survey of the hard corals of the Dampier Archipelago, concentrating on areas not sampled by previous workers. The material reported on herein was collected by the first (DA1/98) of two diving expeditions (DA1/98 and DA3/99) conducted in the Dampier Archipelago as part of the Woodside Energy Ltd/Western Australia Museum partnership.

\section{MATERIALS AND METHODS}

A total of 35 stations was visited in the northeastern region of the Dampier Archipelago, northwestern
Australia, between 17 to 29 October 1998. These consisted of 14 intertidal and 21 subtidal stations. Of the subtidal stations, eight were at depths $>10 \mathrm{~m}$ (refer to Station Lists section in this volume for details). Subtidal stations were examined using SCUBA and intertidal stations were sampled by walking or snorkelling. Usually the stations were discrete habitats and corals were recorded, therefore, from only one habitat type per station. However, some of the intertidal stations covered more than one habitat type, e.g. creek mouths, mud flats and rocky reefs.

The distributions of scleractinian corals were recorded along transects at 20 stations, only one of which was intertidal. A $25 \mathrm{~m}$ long tape was laid out along a depth contour. The transect position was selected haphazardly. A video camera operator filmed the biota along this transect following the method of English et al. (1997). The presence of coral species within $50 \mathrm{~cm}$ either side of these transects was recorded separately. After the transect was completed, corals outside the transect area were examined and any species not found in the transect area were recorded also. These data were kept separate from those collected from the transect. All corals present were recorded regardless of whether they had been found at previous stations. Each station, therefore, had a complete list of corals present. Station 32 was not, however, systematically sampled for hard corals and its list of coral species cannot be considered complete. The video of the transects was analysed by an external organisation (Sinclair Knight Merz Pty. Ltd.) for percentage coverage of the main groups of benthic organisms. The results from this analysis are presented separately (Morrison, this volume). Similarity 
between stations based on the presence/absence of species was analysed using the Ochiai coefficient. Only the transect data were analysed because the area surveyed was the same for each of these stations (Bakus, 1990).

The corals were identified to species. When the coral could not be identified immediately, a sample was collected. Once collected, the hard coral pieces were labelled and placed in a chlorine solution to bleach the skeleton. These were then rinsed in seawater and dried before being packed for transportation to the Western Australian Museum. These samples were identified following Hoeksema (1989), Veron (1986), Veron and Pichon (1976, 1980, 1982), Veron et al. (1977) and Veron and Wallace (1984). The identified specimens have been registered in the collections of the Western Australian Museum.

\section{RESULTS}

A complete list of scleractinian corals present at each station is given in Table 1. A total of 180 zooxanthellate scleractinian species from 47 genera was collected during the present study. Two azooxanthellate scleractinian coral species were collected, i.e. Tubastraea coccinea and T. diaphana (Table 1). Colonies of the species Pocillopora damicornis and Lobophyllia hemprichii were the most ubiquitous present, occurring at 20 stations each (Table 1).

The greatest number of coral species collected at any one station was 50, at intertidal station DA1/98/25 (Keast Island) and subtidal station
DA1/98/13 (8.5 $\mathrm{m}$ depth, Hamersley Shoal). At DA1/98/13, the number of hard coral species per $\mathrm{m}^{2}$ was 1.80 (Table 2). Station DA1/98/29 had the next highest number of coral species per $\mathrm{m}^{2}$ with 1.44. No scleractinian corals were found at stations DA1/98/07 (intertidal, Gidley Island), DA1/98/09 (4.6 m depth, Angel Island) or DA1/98/30 (11.1 m depth, Burrup Peninsula) (Table 2). At all but two transect stations, the percentage of coral species recorded from the transect was $>80 \%$ of those which occurred in the area (Table 2).

A total of 112 species was recorded from the intertidal DA1/98 sites (Appendix 1). Of these, 30 species occurred only at the intertidal stations (Table 3). The most common of these was Euphyllia glabrescens present at six stations, followed by Lobophyllia corymbosa, Montipora turgescens and Platygyra ryukyuensis each recorded from three. At subtidal DA1/98 sites $<10 \mathrm{~m}$ in depth, there was a total of 133 species recorded (Appendix 2). Of these, 30 occurred only at subtidal stations of $<10 \mathrm{~m}$ in depth (Table 4). The most common of these was Montipora danae recorded from three stations. At the subtidal DA1/98 sites $>10 \mathrm{~m}$ in depth, a total of 81 species was recorded (Appendix 3). Of these, 12 species occurred only at subtidal stations $>10 \mathrm{~m}$ in depth (Table 5). Only the azooxanthellate scleractinian Tubastraea diaphana was present at more than one station.

A dendrogram showing the grouping of stations based on the presence or absence of coral species on the transects is presented in Figure 1. Stations DA1/ $98 / 24$ and DA1/98/29 had similar species

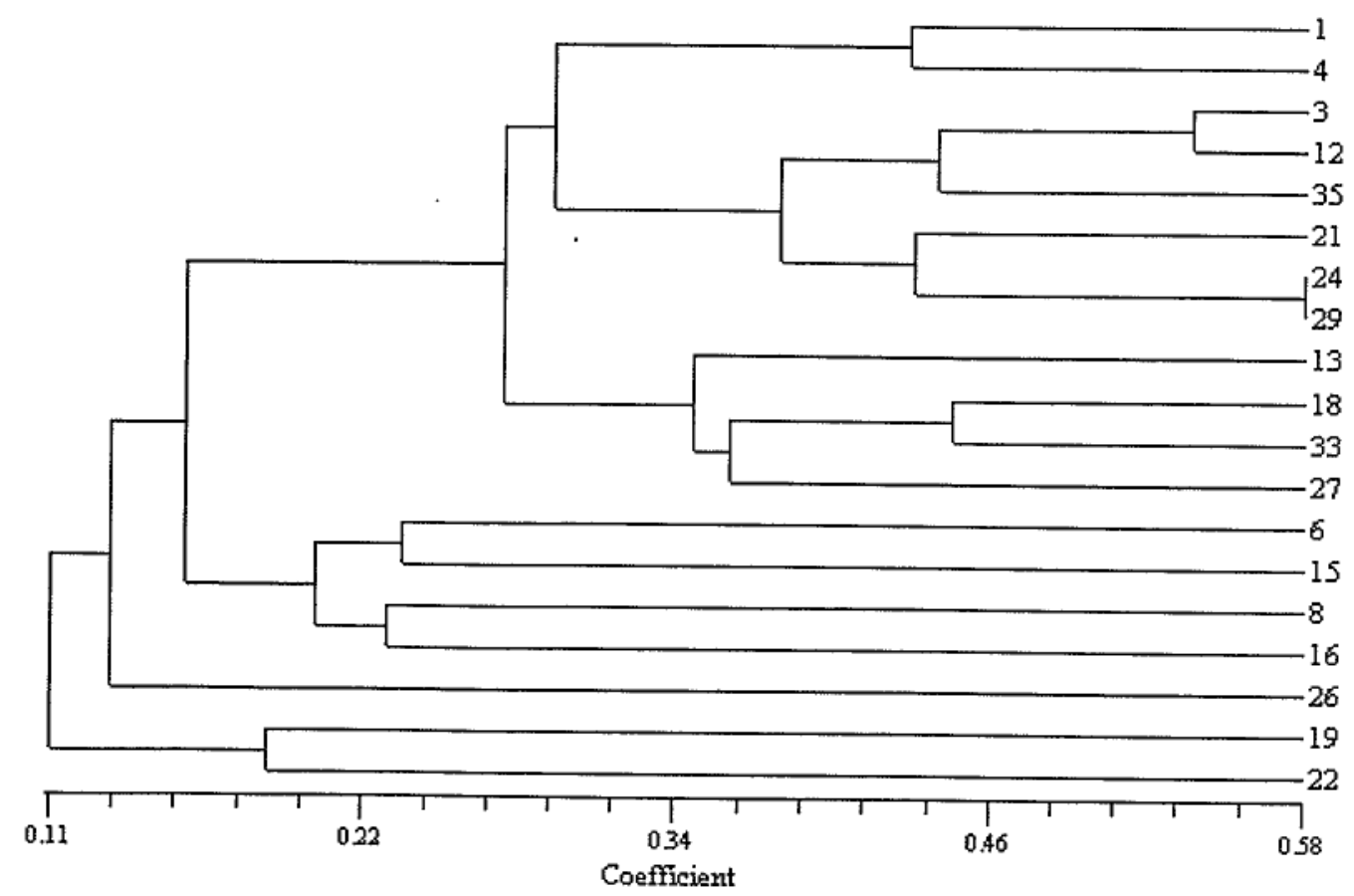

Figure 1 Dendrogram of stations grouped on the basis of species assemblage similarity. 
assemblages, as did DA1/98/3 and DA1/98/12. Each pair had $>50 \%$ similarity in terms of hard coral species present.

One collected colony of Acropora digitifera had large pink gonads. It was collected from intertidal site DA1/98/14 at Unnamed Island $\left(20^{\circ} 26.581 ' S\right.$, $116^{\circ} 48.790^{\prime} \mathrm{E}$ ) on 22 October 1998.

\section{Description of transect stations}

Station DA1/98/01. This station at Dolphin Island had a low relief and was silty. The transect was laid at a depth of $5.7 \mathrm{~m}$. Numerically, this station was dominated by colonies of Porites, Turbinaria and faviid species. Colonies of Acropora and Montipora were less common. Appendix 2 lists the coral species recorded at this station.

Station DA1/98/03. This station at Legendre Island was characterised by deep gullies (2-3 m deep), overhangs, caves and a sandy bottom. The transect was laid at a depth of $7.5 \mathrm{~m}$. Colonies of Acropora were the most dominant numerically and no other coral group was as abundant. Most of these colonies were small (about 15-20 cm diameter). Appendix 2 lists the coral species recorded at this station.

Station DA1/98/04. This station was at Legendre Island and the transect was laid at a depth of $10.9 \mathrm{~m}$. There were many medium-sized colonies of Porites with faviid colonies between them. Appendix 3 lists the coral species recorded at this station.

Station DA1/98/06. This station at Haüy Island had a low relief and was silty. The transect was laid at a depth of $3.2 \mathrm{~m}$. Hard coral coverage was low and the colonies small. No one coral group was dominant. Appendix 2 lists the coral species recorded at this station.

Station DA1/98/08. This station at Angel Island had small gullies of about $0.5 \mathrm{~m}$ depth. The transect was laid at a depth of $3.7 \mathrm{~m}$. The site was dominated numerically and in terms of cover by colonies of plate Acropora. Many of the plates were large ( $>0.5 \mathrm{~m}$ diameter). Appendix 2 lists the coral species recorded at this station.

Station DA1/98/12. This station at Angel Island also had small gullies of about $0.5 \mathrm{~m}$ depth. The transect was laid at a depth of $5.5 \mathrm{~m}$. Hard coral coverage was sparse and there were many dead plates of Acropora covered in silt. These dead plates provided substrata for living hard corals. Colonies of Acropora and faviids were the most numerically dominant coral groups. Appendix 2 lists the coral species recorded at this station.

Station DA1/98/13. This station at Hamersley Shoal was of low relief. The transect was laid at a depth of $8.5 \mathrm{~m}$. Most of the hard coral colonies were encrusting and the colonies small.
Colonies of Acropora were the most dominant numerically, followed by Turbinaria, Montipora and faviid species. Appendix 2 lists the coral species recorded at this station.

Station DA1/98/15. This station at Legendre Island also had a low relief. The transect was laid at a depth of $16.4 \mathrm{~m}$. There were many soft coral colonies present, mainly of a single species of Sinularia. No one group of hard corals was dominant numerically. Appendix 3 lists the coral species recorded at this station.

Station DA1/98/16. This station at Hamersley Shoal was characterised by a low relief and coral rubble. The transect was laid at a depth of 3.5 $\mathrm{m}$. No one group of hard corals was dominant numerically. Appendix 2 lists the coral species recorded at this station.

Station DA1/98/18. This station at Haüy Island had large coral outcrops and Porites bommies separated by patches of sand. The transect was laid at a depth of $11.4 \mathrm{~m}$. Colonies of Acropora, encrusting Montipora and faviids were all common. Appendix 3 lists the coral species recorded at this station.

Station DA1/98/19. This station at Haüy Island was characterised by a low profile reef and silt covered coral rubble. The transect was laid at a depth of $2.7 \mathrm{~m}$. The hard corals consisted of small colonies dominated numerically by Acropora. Appendix 2 lists the coral species recorded at this station.

Station DA1/98/21. This station at Delambre Island had small gullies (about $0.5 \mathrm{~m}$ deep) between outcrops of large colonies of Porites, Pavona and faviids. The transect was laid at a depth of 13.7 $\mathrm{m}$. There were also some large encrusting colonies of Montipora. The most dominant hard corals numerically were faviids and colonies of Pavona, Acropora, Lobophyllia and Montipora. Appendix 3 lists the coral species recorded at this station.

Station DA1/98/22. This station at Delambre Island was characterised by coral rubble and large stands of plate and staghorn Acropora. The transect was laid at a depth of $5.4 \mathrm{~m}$. Appendix 2 lists the coral species recorded at this station.

Station DA1/98/24. This station at Dolphin Island had a relatively low profile and small outcrops of corals. The water was turbid. The transect was laid at a depth of $5.2 \mathrm{~m}$. The site was dominated by colonies of Acropora and faviids. Appendix 2 lists the coral species recorded at this station.

Station DA1/98/26. This station at Madeleine Shoals was a low profile reef dominated by soft corals such as Sarcophyton, Lobophytum, Sinularia and nephtheids. Hard corals were scarce and the most dominant numerically were colonies of Turbinaria. The transect was laid at a depth of 
$21.9 \mathrm{~m}$. Appendix 3 lists the coral species recorded at this station.

Station DA1/98/27. This station at Legendre Island was also a low profile reef. It was silty and had a large population of gorgonians. The transect was laid at a depth of $15.9 \mathrm{~m}$. The hard coral fauna was dominated by colonies of Turbinaria and faviids. Appendix 3 lists the coral species recorded at this station.

Station DA1/98/29. This station at Legendre Island had a high hard coral coverage. There were large stands of staghorn Acropora. In addition to a large proportion of Acropora colonies, Lobophyllia and Fungia were also abundant numerically. The transect was laid at a depth of $4.3 \mathrm{~m}$. Appendix 2 lists the coral species recorded at this station.

Station DA1/98/30. This extremely silty site on the Burrup Peninsula had no hard corals present. The transect was laid at a depth of $11.1 \mathrm{~m}$.

Station DA1/98/32. This station at Legendre Island had a high hard coral coverage and many soft corals. The hard coral fauna was dominated numerically by colonies of Turbinaria and faviids. This site was not sampled systematically for hard coral species but some were collected incidentally. The transect was laid at a depth of $13.6 \mathrm{~m}$. Appendix 3 lists the coral species recorded at this station.

Station DA1/98/33. This station at Angel Island was a low profile reef with much shell grit. The transect was laid at a depth of $8.2 \mathrm{~m}$. The most abundant hard corals were faviids and colonies of Turbinaria. Appendix 2 lists the coral species recorded at this station.

Station DA1/98/35. This station at Legendre Island was the only intertidal station where the corals were recorded from a transect. Colonies of faviids dominated this site. Appendix 1 lists the coral species recorded at this station.

\section{New Records}

Fourteen new records of zooxanthellate scleractinian coral species have been obtained for the Dampier Archipelago (Table 1). These are:

Acanthastrea hillae Wells, 1955 - One specimen was collected from the intertidal station DA1/98/10 $\left(20^{\circ} 28.410^{\prime} \mathrm{S}, 116^{\circ} 48.480^{\prime} \mathrm{E}\right)$ at Angel Island.

Acropora dendrum (Bassett-Smith, 1890) - One specimen was collected from intertidal station DA1/98/17 (20²7.090'S, $\left.116^{\circ} 50.438^{\prime} \mathrm{E}\right)$ at Wilcox Island.

Favites russelli (Wells, 1954) - One specimen was collected from intertidal station DA1/98/25 $\left(20^{\circ} 23.975^{\prime} \mathrm{S}, 116^{\circ} 49.520^{\prime} \mathrm{E}\right)$ at Keast Island.

Fungia (Lobactis) scutaria Lamarck, 1801 - One specimen was collected from a depth of $8.5 \mathrm{~m}$ at station DA1/98/13 (20²3.203'S, $\left.116^{\circ} 46.691^{\prime} \mathrm{E}\right)$ at Hamersley Shoal.
Goniopora pandoraensis Veron and Pichon, 1982 One specimen was collected from a depth of 8.5 $\mathrm{m}$ at station DA1/98/13 (20²3.203'S, $\left.116^{\circ} 46.691^{\prime} \mathrm{E}\right)$ at Hamersley Shoal.

Leptastrea transversa Klunzinger, 1879 - One specimen each was collected from intertidal stations DA1/98/05 (20²5.380'S, 116 $\left.57.510^{\prime} \mathrm{E}\right)$ at Legendre Island and DA1/98/10 $\left(20^{\circ} 28.410^{\prime} \mathrm{S}, 116^{\circ} 48.480^{\prime} \mathrm{E}\right)$ at Angel Island.

Montipora caliculata (Dana, 1846) - One specimen each was collected from station DA1/98/19 $\left(20^{\circ} 26.620^{\prime} \mathrm{S}, 116^{\circ} 58.390^{\prime} \mathrm{E}\right)$ at a depth of $2.7 \mathrm{~m}$ at Haüy Island and intertidal station DA1/98/28 $\left(20^{\circ} 25.771 ' \mathrm{~S}, 116^{\circ} 52.680^{\prime} \mathrm{E}\right)$ at Dolphin Island.

Montipora capricornis Veron, 1985 - One specimen each was collected from station DA1/98/06 $\left(20^{\circ} 25.725^{\prime} \mathrm{S}, 116^{\circ} 57.580^{\prime} \mathrm{E}\right)$ at $3.2 \mathrm{~m}$ depth at Haüy Island and intertidal station DA1/98/25 $\left(20^{\circ} 23.975^{\prime} \mathrm{S}, 116^{\circ} 49.520^{\prime} \mathrm{E}\right)$ at Keast Island.

Montipora foliosa (Pallas, 1766) - One specimen each was collected from station DA1/98/18 $\left(20^{\circ} 26.400^{\prime} \mathrm{S}, 116^{\circ} 58.634^{\prime} \mathrm{E}\right)$ at $11.4 \mathrm{~m}$ depth at Haüy Island and station DA1/98/29 $\left(20^{\circ} 24.566^{\prime} \mathrm{S}, 116^{\circ} 53.714^{\prime} \mathrm{E}\right)$ at $4.3 \mathrm{~m}$ at Legendre Island.

Montipora informis Bernard, 1897 - One specimen each was collected from station DA1/98/03 $\left(20^{\circ} 24.320^{\prime} \mathrm{S}, 116^{\circ} 56.108^{\prime} \mathrm{E}\right)$ at $7.5 \mathrm{~m}$ depth at Legendre Island and station DA1/98/06 $\left(20^{\circ} 25.725^{\prime} \mathrm{S}, 116^{\circ} 57.580^{\prime} \mathrm{E}\right)$ at $3.2 \mathrm{~m}$ depth at Haüy Island.

Montipora stellata Bernard, 1897 - One specimen each was collected from station DA1/98/01 $\left(20^{\circ} 25.852^{\prime} \mathrm{S}, 116^{\circ} 52.953^{\prime} \mathrm{E}\right)$ at $5.7 \mathrm{~m}$ depth at Dolphin Island and station DA1/98/18 $\left(20^{\circ} 26.400^{\prime} \mathrm{S}, 116^{\circ} 58.634^{\prime} \mathrm{E}\right)$ at $11.4 \mathrm{~m}$ depth at Haüy Island.

Montipora venosa (Ehrenberg, 1834) - One specimen was collected from intertidal station DA1/98/ $17\left(20^{\circ} 27.090^{\prime} \mathrm{S}, 116^{\circ} 50.438^{\prime} \mathrm{E}\right)$ at Wilcox Island.

Pavona clavus (Forskål, 1775) - One specimen was collected from station DA1/98/04 (20²4.320'S, $\left.116^{\circ} 56.108^{\prime} \mathrm{E}\right)$ at $10.9 \mathrm{~m}$ depth at Legendre Island.

Seriatopora caliendrum (Ehrenberg, 1834)- One specimen was collected from station DA1/98/ $29\left(20^{\circ} 24.566^{\prime} \mathrm{S}, 116^{\circ} 53.714^{\prime} \mathrm{E}\right)$ at $4.3 \mathrm{~m}$ at Legendre Island.

\section{DISCUSSION}

The number of hard coral species recorded at each station ranged from 0 to 50 (Table 2). This is similar to the range that Marsh (1978) reported from her survey. In her study, the number of coral species per station ranged from 7 to 55. A complete updated list of zooxanthellate scleractinian corals recorded from the Dampier Archipelago is presented in Appendix 4. The total number of 
zooxanthellate scleractinian coral species now known from the Dampier Archipelago is 229 from 57 genera. This places the Dampier Archipelago second only to Ashmore Reef in terms of the number of coral species recorded from Western Australia (Veron, 1993).

The new records from this study that represent northerly extensions of the coral's known range within Western Australian waters were Acanthastrea hillae, Acropora dendrum and Montipora capricornis (Veron and Marsh, 1988). The new records that represented a southerly extension of the coral's known range within Western Australian waters were Goniopora pandoraensis and Pavona clavus (Veron and Marsh, 1988). The following coral species had all been collected previously further north and south of the Dampier Archipelago (Veron. and Marsh, 1988): Favites russelli, Fungia scutaria, Leptastrea transversa, $M$. caliculata, $M$. foliosa, $M$. informis, $M$. stellata, $M$. venosa and Seriatopora caliendrum. Colonies of $M$. stellata and $M$. venosa had also been collected from the Montebello Islands just west of the Dampier Archipelago. Most of the new records for the Dampier Archipelago were from those sites around Legendre, Haüy and Keast Islands and Hamersley Shoal, i.e. the outer areas of the archipelago. Only six of the sites in this study overlapped with those surveyed by previous workers. Stations 8,31 and 35 were close to three sites of Marsh (1978) (see Paling, 1986 for a map of Marsh's stations) and stations 13, 16 and 32 were similar to those of Simpson (1988). Thus, not only did this study survey many areas different to where Marsh (1978) and Simpson (1988) conducted fieldwork, but it also examined sites that were deeper than $10 \mathrm{~m}$. In view of the known ranges of these coral species and where they were collected, these new records for the Dampier Archipelago are not surprising.

A complete, updated list of azooxanthellate scleractinian corals recorded from the Dampier Archipelago is presented in Appendix 5. No new records were reported for these corals from this survey. As this group of corals was not targeted during the survey, this result is to be expected.

The subtidal station with the highest number of species recorded (station DA1/98/13, Table 2) did not have the highest hard coral coverage (see results section on transect descriptions). Luxuriant hard coral growth does not necessarily mean high species diversity. Also, interestingly, the station with the biggest gullies and, therefore, the most habitat complexity (station DA1/98/03) only had 31 species recorded from it. In contrast, station DA1/98/13 was a low profile reef. The colonies at DA1/98/13 were small which indicates that they are relatively young. This site, being relatively exposed, would be subjected to periodic violent storms. Colonies would, therefore, be prevented from attaining their maximum size and outcompeting other species. This is in keeping with the intermediate disturbance hypothesis of Connell (1978).

When Paling (1986) analysed the data of Marsh (1978), he found that the protected sites on the Burrup Peninsula had less species richness and hard coral coverage than the more exposed sites. The results of the present study did not conform to this trend. For example, station DA1/98/15 at Cape Legendre, a very exposed site, only had four hard coral species present. It did, however, have many soft corals. Species indicative of clear water such as Pocillopora eydouxi (Table 1) occurred around the seaward parts of Legendre and Delambre Islands and at Hamersley Shoal (at stations DA1/98/04, DA1/98/13, DA1/98/16 and DA1/98/21). Although one of these stations had the highest number of species recorded for this study, the others fell into the middle range of species richness. Marsh (1978) also observed that turbidity did not necessarily lower species richness or hard coral coverage.

Neither Marsh (1978) nor Paling (1986) could discern any coral assemblages that denoted a habitat type. This corresponds with the results of the present study when the stations were analysed in terms of species assemblage similarity (Figure 1). Most stations were different from each other and only two pairs clustered together. Stations DA1/ $98 / 24$ and DA1/98/29 had $>50 \%$ of species in common. Both were located in Nickol Bay and were shallow sites (5.2 m and $4.3 \mathrm{~m}$ depth respectively). However, from the descriptions of the transect stations (see Results) they were very different in terms of coral cover. A similar situation occurred for the other two stations that had $>50 \%$ of species in common, namely DA1/98/03 and DA1/98/12. Station DA1/98/03 had a good hard coral coverage whereas station DA1/98/12 was sparse. The only possible link is that both sites were located on the outer seaward edge of islands. However, none of the other sites located nearby grouped with this pair. This analysis indicates that an estimation of the abundance of each species present is also needed to obtain a more complete understanding of the ecological regimes that govern species' distributions.

The single colony of Acropora digitifera observed with mature ova would have been ready to spawn in November or December. In a review of coral spawning (Fadlallah, 1983), colonies of $A$. digitifera on the Great Barrier Reef, Australia, broadcast their spawn during spring or early summer. However, many corals on the west coast of Australia spawn in early autumn (March-April) (Simpson, 1988). When Simpson sampled hard coral colonies in November 1984 and October 1985, he found no mature ova. $\mathrm{He}$ did not, however, sample any colonies of $A$. 
digitifera. This is the first report of a hard coral colony on the west coast of Australia containing mature ova during spring (October).

In conclusion, the Dampier Archipelago has a rich coral fauna despite extremely turbid waters. Generally, the corals were healthy and some areas showed profuse growth. The transects provided an accurate representation of the fauna present in the area and this method should be expanded upon in future to allow for a more detailed analysis of the coral community.

\section{ACKNOWLEDGEMENTS}

I wish to thank $M$. Cowan for conducting the statistical analysis of the similarity of stations; $M$. Vanderklift and P. Morrison for videoing the transects; J. Ralston and crew for their help aboard the Kimberley Quest, and J. Fromont, M. Hewitt, S. Morrison, C. Bryce and S. Slack-Smith for their help during fieldwork.

\section{REFERENCES}

Bakus, G.J. (1990). Quantitative Ecology and Marine Biology. Oxford \& IBH Publishing Co. Pvt. Ltd., New Delhi.

Cairns, S.D. (1998). Azooxanthellate Scleractinia (Cnidaria: Anthozoa) of Western Australia. Records of the Western Australian Museum 18: 361-417.

Connell, J.H. (1978). Diversity in tropical rain forests and coral reefs. Science 199: 1302-1309.

English, S., Wilkinson, C. and Baker, V. (eds) 1997. Survey Manual for Tropical Marine Resources. (Second edition). Australian Institute of Marine Science, Townsville.

Fadlallah, Y.H. (1983). Sexual reproduction, development and larval biology in scleractinian corals: A review. Coral Reefs 2: 129-150.

Hoeksema, B.W. (1989). Taxonomy, phylogeny and biogeography of mushroom corals (Scleractinia: Fungiidae). Zoologische Verhandelingen 254: 1-295.
Marsh, L.M. (1978). Report on the corals and some associated invertebrates of the Dampier Archipelago: 1-67. In Hutchins, J.B., Slack-Smith, S.M. and Marsh, L.M. (eds), Report on the Marine Fauna and Flora of the Dampier Archipelago. Western Australian Museum, Perth. Unpublished reports submitted to Meagher \& LeProvost, Consultant Biologists, December, 1978.

Simpson, C. (1988). Ecology of scleractinian corals in the Dampier Archipelago, Western Australia. Technical Series 23: 1-238. Environmental Protection Authority, Perth, Western Australia.

Veron, J.E.N. (1986). Corals of Australia and the Indo-Pacific. Angus \& Robertson, Sydney.

Veron, J.E.N. (1993). A biogeographic database of hermatypic corals: species of the central Indo-Pacific, genera of the world. Australian Institute of Marine Science, Monograph Series 10: 1-433.

Veron, J.E.N. and Marsh, L.M. (1988). Hermatypic corals of Western Australia: records and annotated species list. Records of the Western Australian Museum, Supplement 29: 1-136.

Veron, J.E.N. and Pichon, M. (1976). Scleractinia of eastern Australia. Part I. Families Thamnasteriidae, Astrocoeniidae, Pocilloporidae. Australian Institute of Marine Science Monograph Series 1: 1-86.

Veron, J.E.N. and Pichon, M. (1980). Scleractinia of eastern Australia. Part III. Families Agariciidae, Siderastreidae, Fungiidae, Oculinidae, Merulinidae, Mussidae, Pectiniidae, Caryophylliidae, Dendrophylliidae. Australian Institute of Marine Science Monograph Series 4: 1-443.

Veron, J.E.N. and Pichon, M. (1982). Scleractinia of eastern Australia. Part IV. Family Poritidae. Australian Institute of Marine Science Monograph Series 5: 1-159.

Veron, J.E.N., Pichon, M. and Wijsman-Best, M. (1977). Scleractinia of eastern Australia. Part II. Families Faviidae, Trachyphylliidae. Australian Institute of Marine Science Monograph Series 3: 1-233.

Veron, J.E.N. and Wallace, C.C. (1984). Scleractinia of eastern Australia. Part V. Family Acroporidae. Australian Institute of Marine Science Monograph Series 6: 1-485. 
Table 1 A list of scleractinian corals recorded from the Dampier Archipelago during October 1998. C - sample was collected from an area other than from a transect; $\mathrm{V}$ - visual record only; $\mathrm{T}$ - coral was recorded from a transect.

\begin{tabular}{|c|c|c|c|}
\hline Taxa & DA1/98 Station Numbers & Taxa & DA1/98 Station Numbers \\
\hline Family Acroporidae & & Montipora mollis & $6 \mathrm{~T}, 13 \mathrm{~T}, 25 \mathrm{C}, 33 \mathrm{~T}, 34 \mathrm{C}$ \\
\hline Acropora aculeus & $1 \mathrm{~T}, 13 \mathrm{~T}, 21 \mathrm{~T}, 27 \mathrm{TC}$ & Montipora monasteriata & $3 \mathrm{~T}, 13 \mathrm{~T}, 18$ \\
\hline Acropora acuminata & $18 \mathrm{~T}$ & Montipora peltiformis & $13 \mathrm{~T}$ \\
\hline Acropora anthocercis & $23 \mathrm{C}$ & Montipora spongodes & $17 \mathrm{C}, 31 \mathrm{C}$ \\
\hline Acropora aspera & $31 \mathrm{C}$ & Montipora spumosa & $29 \mathrm{~T}$ \\
\hline Acropora austera & $17 \mathrm{C}, 22 \mathrm{~T}, 25 \mathrm{C}$ & Montipora stellata & $1 \mathrm{~T}, 18 \mathrm{~T}$ \\
\hline Acropora cerealis & $13 \mathrm{~T}, 21 \mathrm{~T}$ & Montipora tuberculosa & $21 \mathrm{~T}, 31 \mathrm{C}$ \\
\hline Acropora clathrata & $13 \mathrm{~T}, 22 \mathrm{~T}$ & Montipora turgescens & $20 \mathrm{C}, 25 \mathrm{C}, 31 \mathrm{C}$ \\
\hline Acropora cytherea & $\begin{array}{l}8 \mathrm{~T}, 12 \mathrm{~T}, 13 \mathrm{~T}, 16 \mathrm{C}, 19 \mathrm{~T}, 22 \mathrm{~T}, \\
25 \mathrm{C}\end{array}$ & $\begin{array}{l}\text { Montipora turtlensis } \\
\text { Montipora undata }\end{array}$ & $\begin{array}{l}4 \mathrm{~T} \\
13 \mathrm{~T}\end{array}$ \\
\hline Acropora danai & $22 \mathrm{~T}$ & Montipora venosa & $17 \mathrm{C}$ \\
\hline Acropora dendrum & $17 \mathrm{C}$ & Montipora verrucosa & $4 \mathrm{~T}$ \\
\hline Acropora digitifera & $\begin{array}{l}3 \mathrm{C}, 6 \mathrm{C}, 10 \mathrm{C}, 14 \mathrm{C}, 19 \mathrm{~T}, 20 \mathrm{C} \\
23 \mathrm{C}, 25 \mathrm{C}, 31 \mathrm{C}\end{array}$ & Family Agariciidae & \\
\hline Acropora divaricata & $8 \mathrm{~T}, 17 \mathrm{C}$ & lser & \\
\hline Acropora florida & $1 \mathrm{TV}, 4 \mathrm{TV}, 12 \mathrm{~T}, 13 \mathrm{~V}, 24 \mathrm{~T}$ & $\begin{array}{l}\text { Pachyserls speciosa } \\
\text { Pavona clavus }\end{array}$ & $\begin{array}{l}3 \mathrm{~T}, 15 \mathrm{~T}, 27 \mathrm{C} \\
4 \mathrm{~T}\end{array}$ \\
\hline Acropora formosa & $13 \mathrm{~T}, 17 \mathrm{C}, 19 \mathrm{~T}, 24 \mathrm{C}, 34 \mathrm{C}$ & Pavona decussata & $3 \mathrm{~T}, 4 \mathrm{~T}, 12 \mathrm{TV}, 13 \mathrm{TV}, 20 \mathrm{~V}$, \\
\hline Acropora gemmifera & $14 \mathrm{C}$ & & 21TV, 24TV, 25V, 29C, 35C \\
\hline Acropora humilis & $10 \mathrm{C}, 16 \mathrm{~T}, 24 \mathrm{C}, 31 \mathrm{C}$ & Pavona varians & $4 \mathrm{~T}$ \\
\hline Acropora hyacinthus & $\begin{array}{l}3 \mathrm{~T}, 5 \mathrm{C}, 8 \mathrm{~T}, 16 \mathrm{~T}, 23 \mathrm{C}, 25 \mathrm{C}, \\
31 \mathrm{C}\end{array}$ & Family Astrocoeniidae & \\
\hline Acropora latistella & $4 \mathrm{~T}, 16 \mathrm{C}, 23 \mathrm{C}, 24 \mathrm{~T}, 25 \mathrm{C}$ & Stylocoeniella guentheri & $20 \mathrm{C}, 21 \mathrm{~T}$ \\
\hline Acropora microphthalma & $29 \mathrm{~T}$ & Family Caryophylliidae & \\
\hline Acropora millepora & $10 \mathrm{C}, 29 \mathrm{~T}, 31 \mathrm{C}$ & aphyll & $31 \mathrm{C}$ \\
\hline Acropora nasuta & $\begin{array}{c}3 \mathrm{~T}, 4 \mathrm{C}, 6 \mathrm{C}, 12 \mathrm{~T}, 18 \mathrm{~T}, 19 \mathrm{~T} \\
21 \mathrm{~T}, 22 \mathrm{~T}, 24 \mathrm{~T}, 25 \mathrm{C}, 29 \mathrm{~T}\end{array}$ & $\begin{array}{l}\text { Euphyllia ancora } \\
\text { Euphyllia divisa }\end{array}$ & $\begin{array}{l}33 \mathrm{~T} \\
10 \mathrm{C}\end{array}$ \\
\hline Acropora nobilis & $\begin{array}{l}3 \mathrm{~T}, 12 \mathrm{~T}, 13 \mathrm{~T}, 22 \mathrm{~T}, 25 \mathrm{C}, 29 \mathrm{~T} \text {, } \\
\quad 32 \mathrm{C}\end{array}$ & Euphyllia glabrescens & $11 \mathrm{C}, 17 \mathrm{~V}, 25 \mathrm{~V}, 28 \mathrm{~V}, 31 \mathrm{~V}, 34 \mathrm{~V}$ \\
\hline Acropora polystoma & $12 \mathrm{~T}$ & Family Dendrophylliidae & \\
\hline Acropora & 8 & Duncanopsammia axifuga & $13 \mathrm{C}$ \\
\hline Acropora & $29 \mathrm{~T}, 31 \mathrm{C}$ & Tubastraea coccinea & $3 C$ \\
\hline Acropora & $27,, 01$ & Tubastraea diaphana & $15 \mathrm{TC}, 26 \mathrm{~T}$ \\
\hline Acropora & $29 \mathrm{~T}$ & Turbinaria bifrons & $6 \mathrm{~T}, 10 \mathrm{C}, 11 \mathrm{C}, 14 \mathrm{C}, 17 \mathrm{~V}, 25 \mathrm{C}$ \\
\hline Acropora selago & 1 & Turbinaria conspicua & $14 \mathrm{C}, 33 \mathrm{~T}$ \\
\hline Acropora solitaryensis & $4 \mathrm{~T}, 8 \mathrm{~T}$ & Turbinaria frondens & $1 \mathrm{C}, 8 \mathrm{C}, 13 \mathrm{~T}, 18 \mathrm{~T}, 24 \mathrm{~T}, 32 \mathrm{TV}$, \\
\hline Acropora spicifera & $8 \mathrm{~T}, 13 \mathrm{~T}, 25 \mathrm{C}$ & & $33 \mathrm{~T}$ \\
\hline Acropora subulata & $13 \mathrm{~T}$ & Turbinaria mesenterina & 1T, 4TC \\
\hline Acropora tenuis & $\begin{array}{l}14 \mathrm{C}, 17 \mathrm{C}, 19 \mathrm{~T}, 21 \mathrm{~T}, 24 \mathrm{~T}, 29 \mathrm{~T} \\
35 \mathrm{~T}\end{array}$ & Turbinaria peltata & $\begin{array}{c}4 \mathrm{TC}, 6 \mathrm{TC}, 10 \mathrm{C}, 13 \mathrm{~T}, 16 \mathrm{C}, \\
26 \mathrm{~T}, 2 \mathrm{~T}, 31 \mathrm{C}, 32 \mathrm{TV}, 33 \mathrm{~T}\end{array}$ \\
\hline Acropora valenciennesi & $18 \mathrm{~T}$ & Turbinaria reniformis & $1 \mathrm{~T}, 3 \mathrm{~T}, 4 \mathrm{~T}, 6 \mathrm{~T}, 12 \mathrm{~T}, 15 \mathrm{~T}, 16 \mathrm{~T}$, \\
\hline Acropora valida & $\begin{array}{l}18 \mathrm{~T}, 19 \mathrm{~T}, 20 \mathrm{C}, 24 \mathrm{~T}, 25 \mathrm{C}, 29 \mathrm{~T} \\
31 \mathrm{C}\end{array}$ & & $\begin{array}{l}17 \mathrm{~V}, 20 \mathrm{C}, 23 \mathrm{C}, 24 \mathrm{~T}, 26 \mathrm{TV} \\
27 \mathrm{~T}, 31 \mathrm{~V}, 32 \mathrm{TV}, 35 \mathrm{~V}\end{array}$ \\
\hline Acropora vaughani & $31 \mathrm{C}$ & Turbinaria stellulata & $12 \mathrm{~T}, 16 \mathrm{~T}, 27 \mathrm{~T}$ \\
\hline Acropora verweyi & $24 \mathrm{C}, 35 \mathrm{C}$ & Fan & \\
\hline Acropora yongei & $8 \mathrm{~T}$ & Barabattoia amicorum & $1 \mathrm{~T}, 2 \mathrm{C}, 18 \mathrm{~T}, 24 \mathrm{~T}, 25 \mathrm{C}, 27 \mathrm{~T}$, \\
\hline Astreopora explanata & $20 \mathrm{C}$ & & $33 \mathrm{~T}$ \\
\hline Astreopora gracilis & $1 \mathrm{~T}$ & Caulastrea tumida & $1 \mathrm{~T}, 10 \mathrm{C}, 14 \mathrm{~V}, 17 \mathrm{~V}, 20 \mathrm{~V}, 23 \mathrm{~V}$ \\
\hline Astreopora myriophthalma & $\begin{array}{l}4 \mathrm{~T}, 12 \mathrm{~T}, 13 \mathrm{TV}, 18 \mathrm{TV}, 24 \mathrm{~T} \\
25 \mathrm{~V}, 27 \mathrm{~T}, 33 \mathrm{~T}\end{array}$ & & $\begin{array}{l}24 \mathrm{TV}, 25 \mathrm{~V}, 28 \mathrm{~V}, 29 \mathrm{TV}, \\
31 \mathrm{~V}, 34 \mathrm{~V}, 35 \mathrm{TV}\end{array}$ \\
\hline Montipora aequituberculata & $19 \mathrm{~T}, 29 \mathrm{~T}, 34 \mathrm{C}$ & Cyphastrea microphthalma & $1 \mathrm{~T}, 3 \mathrm{~T}, 12 \mathrm{~T}, 18 \mathrm{~T}, 19 \mathrm{~T}, 20 \mathrm{C}$ \\
\hline Montipora caliculata & $19 \mathrm{~T}, 28 \mathrm{C}$ & & $21 \mathrm{~T}, 24 \mathrm{TV}, 35 \mathrm{~T}$ \\
\hline Montipora capricornis & $6 \mathrm{~T}, 25 \mathrm{C}$ & Cyphastrea serailia & $6 \mathrm{~T}, 14 \mathrm{C}, 16 \mathrm{~T}, 20 \mathrm{C}, 25 \mathrm{C}, 31 \mathrm{C}$, \\
\hline Montipora crassituberculata & $21 \mathrm{~T}$ & & $33 \mathrm{~T}$ \\
\hline Montipora danae & $8 \mathrm{~T}, 12 \mathrm{~T}, 13 \mathrm{~T}$ & Echinopora horrida & $4 \mathrm{~T}$ \\
\hline Montipora efflorescens & $20 \mathrm{C}, 21 \mathrm{~T}, 29 \mathrm{~T}$ & Echinopora lamellosa & $18 \mathrm{~T}, 20 \mathrm{C}, 29 \mathrm{~T}$ \\
\hline Montipora foliosa & $18 \mathrm{~T}, 29 \mathrm{~T}$ & Favia favus & $4 \mathrm{TC}, 8 \mathrm{~T}, 12 \mathrm{~T}, 14 \mathrm{~V}, 18 \mathrm{~V}, 20 \mathrm{C}$, \\
\hline Montipora foveolata & $31 \mathrm{C}$ & & $23 \mathrm{C}, 25 \mathrm{~V}, 26 \mathrm{~T}, 29 \mathrm{TV}, 31 \mathrm{~V}$ \\
\hline Montipora grisea & $29 \mathrm{~T}$ & & $33 \mathrm{TV}, 35 \mathrm{TV}$ \\
\hline Montipora hispida & $19 \mathrm{~T}, 20 \mathrm{C}, 26 \mathrm{~T}$, & Favia lizardensis & $10 \mathrm{C}$ \\
\hline & & Favia matthaii & $18 \mathrm{~T}, 1$ \\
\hline Montipora hoffmeisteri & $\begin{array}{l}13 \mathrm{~T}, 18 \mathrm{~T}, 19 \mathrm{~T}, 29 \mathrm{~T} \\
3 \mathrm{~T}, 6 \mathrm{C}\end{array}$ & Favia pallida & $3 \mathrm{~T}, 12 \mathrm{~T}, 13 \mathrm{~T}, 14 \mathrm{C}, 16 \mathrm{C}, 19 \mathrm{~T}$, \\
\hline $\begin{array}{l}\text { Montipora informis } \\
\text { Montipora millepora }\end{array}$ & $\begin{array}{l}3 \mathrm{~T}, 6 \mathrm{C} \\
1 \mathrm{~T}, 2 \mathrm{~T}\end{array}$ & Favia rotumana & $6 \mathrm{C} ; 8 \mathrm{~T}$ \\
\hline
\end{tabular}


Table 1 (cont.)

\begin{tabular}{ll|ll}
\hline \multicolumn{1}{c|}{ Taxa } & DA1/98 Station Numbers & \multicolumn{1}{c}{ Taxa } & DA1/98 Station Numbers \\
\hline Favia rotundata & $1 \mathrm{~T}, 10 \mathrm{C}, 11 \mathrm{C}, 17 \mathrm{C}, 21 \mathrm{~T}, 28 \mathrm{C}$, & Lobophyllia corymbosa & 20C, 25V, 31V \\
Favia speciosa & $31 \mathrm{C}$ & Lobophyllia hataii & $4 \mathrm{C}, 13 \mathrm{~T}$ \\
Favia stelligera & $17 \mathrm{C}$ & Lobophyllia hemprichii & $1 \mathrm{~T}, 3 \mathrm{TV}, 4 \mathrm{~T}, 10 \mathrm{C}, 12 \mathrm{TV}$, \\
Favia veroni & $3 \mathrm{~T}$ & & $13 \mathrm{TV}, 14 \mathrm{~V}, 15 \mathrm{~V}, 17 \mathrm{~V}$, \\
Favites abdita & $17 \mathrm{C}$ & $18 \mathrm{TV}, 20 \mathrm{C}, 21 \mathrm{TV}, 22 \mathrm{TV}$, \\
& $1 \mathrm{~T}, 3 \mathrm{~T}, 4 \mathrm{~T}, 12 \mathrm{~T}, 14 \mathrm{~V}, 18 \mathrm{~T}$, & & $23 \mathrm{C}, 24 \mathrm{TV}, 25 \mathrm{~V}, 29 \mathrm{TV}$, \\
& $20 \mathrm{C}, 21 \mathrm{~T}, 24 \mathrm{~T}, 29 \mathrm{TV}, 33 \mathrm{TV}$, & & $31 \mathrm{C}, 33 \mathrm{TV}, 35 \mathrm{TV}$ \\
Favites chinensis & $34 \mathrm{~V}, 35 \mathrm{TV}$ & Symphyllia agaricia & $13 \mathrm{TV}$ \\
Favites complanata & $2 \mathrm{C}, 8 \mathrm{~T}, 16 \mathrm{~T}$ & Symphyllia recta & $3 \mathrm{~T}, 8 \mathrm{~T}, 27 \mathrm{TV}, 32 \mathrm{TV}$
\end{tabular}

Favites flexuosa

Favites halicora

Favites pentagona

Favites russelli

Goniastrea aspera

Goniastrea australensis

Goniastrea edwardsi

Goniastrea favulus

Goniastrea palauensis

Goniastrea pectinata

Goniastrea retiformis

Hydnophora exesa

Hydnophora microconos

Hydnophora pilosa

Hydnophora rigida

Leptastrea purpurea

Leptastrea transversa

Leptoria phrygia

Montastrea curta

Montastrea magnistellata

Montastrea valenciennesi

Moseleya latistellata

Oulophyllia bernettae

Platygyra daedalea

Platygyra lamellina

Platygyra pini

Platygyra ryukyuensis

Platygyra sinensis

Platygyra verweyi

Plesiastrea versipora

Family Fungidae

Fungia cyclolites

Fungia fungites

$10 \mathrm{C}, 12 \mathrm{~T}, 13 \mathrm{~T}, 14 \mathrm{C}, 21 \mathrm{~T}, 25 \mathrm{C}$, $31 \mathrm{C}$

2C, $13 \mathrm{~T}, 25 \mathrm{C}$

$4 \mathrm{~T}, 10 \mathrm{C}, 16 \mathrm{~T}, 19 \mathrm{~T}$

$25 \mathrm{C}$

2C, 5V, 6TC, 8T, 10C, 11C, $12 \mathrm{~T}, 13 \mathrm{~T}, 17 \mathrm{C}, 20 \mathrm{C}, 23 \mathrm{C}$, $28 \mathrm{C}, 31 \mathrm{C}$

$14 \mathrm{~V}, 24 \mathrm{TV}$

$8 \mathrm{~T}$

3T, 18T, 20C, 21T

$4 \mathrm{~T}, 16 \mathrm{~T}, 25 \mathrm{C}$

$1 \mathrm{TC}, 13 \mathrm{~T}, 19 \mathrm{C}, 21 \mathrm{~T}, 27 \mathrm{~T}$

$3 \mathrm{~T}, 8 \mathrm{~T}, 12 \mathrm{~T}, 20 \mathrm{C}, 25 \mathrm{C}, 35 \mathrm{TV}$

13T, 18T, 19TV, 25C, 33TV

$13 \mathrm{~T}, 14 \mathrm{C}$

$10 \mathrm{C}, 20 \mathrm{C}$

$24 \mathrm{~T}, 29 \mathrm{TV}$

$10 \mathrm{C}$

$5 \mathrm{C}, 10 \mathrm{C}$

12T, 25V, 33TV, 25TV

$3 \mathrm{~T}, 4 \mathrm{~T}, 6 \mathrm{C}, 12 \mathrm{C}, 18 \mathrm{~T}$

13T, 19T, 20C, 29TV

$1 \mathrm{~T}, 4 \mathrm{~T}, 10 \mathrm{C}, 20 \mathrm{C}, 27 \mathrm{~T}$

$4 \mathrm{TC}, 10 \mathrm{C}, 25 \mathrm{C}, 27 \mathrm{TV}, 31 \mathrm{C}$, $33 T V$

$16 \mathrm{~T}, 20 \mathrm{C}$

$3 \mathrm{~T}, 6 \mathrm{C}, 8 \mathrm{~T}, 10 \mathrm{C}, 12 \mathrm{~T}, 13 \mathrm{~T}$, $16 \mathrm{~T}, 18 \mathrm{~T}, 20 \mathrm{C}, 23 \mathrm{C}, 25 \mathrm{C}$, 27T, 33TV

$20 \mathrm{C}, 31 \mathrm{C}$

1T, 4TC, 8T, 15T, 18T

$10 \mathrm{C}, 20 \mathrm{C}, 25 \mathrm{C}$

$3 \mathrm{~T}, 5 \mathrm{C}, 6 \mathrm{~T}, 8 \mathrm{~T}, 10 \mathrm{C}, 12 \mathrm{~T}, 14 \mathrm{C}$, $19 \mathrm{~T}, 25 \mathrm{C}$

$5 \mathrm{C}$

Symphyllia recta

Family Oculinidae

Galaxea astreata

Galaxea fascicularis

Family Pectiniidae

Echinophyllia aspera

Mycedium elephantotus

Oxypora lacera

Pectinia lactuca

Pectinia paeonia

Family Pocilloporidae

Pocillopora damicornis

$6 \mathrm{~T}, 12 \mathrm{~T}, 14 \mathrm{C}, 16 \mathrm{C}, 23 \mathrm{~V}, 25 \mathrm{~V}$, $31 \mathrm{~V}, 35 \mathrm{TV}$

$31 \mathrm{C}$

$1 \mathrm{~T}, 4 \mathrm{C}, 10 \mathrm{C}, 12 \mathrm{C}, 13 \mathrm{TC}, 14 \mathrm{C}$, $17 \mathrm{C}, 18 \mathrm{~T}, 20 \mathrm{C}, 21 \mathrm{~T}, 24 \mathrm{~V}$, 25C, 29TV, 31C, 35TC $13 \mathrm{C}$

Fungia scutaria

Herpolitha limax

Podabacia crustacea Polyphyllia talpina

Family Merulinidae Merulina ampliata

Family Mussidae Acanthastrea echinata

Acanthastrea hillae

Australomussa rowleyensis

$1 \mathrm{~T}, 10 \mathrm{~V}, 13 \mathrm{~T}, 17 \mathrm{C}, 19 \mathrm{~T}, 20 \mathrm{C}$, $24 \mathrm{~T}, 25 \mathrm{~V}, 29 \mathrm{TV}, 32 \mathrm{C}, 33 \mathrm{~T}$

$3 \mathrm{~T}, 4 \mathrm{C}, 12 \mathrm{~T}, 20 \mathrm{C}$

13TV, $27 \mathrm{~T}$

$3 \mathrm{~T}, 12 \mathrm{~T}, 13 \mathrm{TV}, 17 \mathrm{~V}, 20 \mathrm{C}$ $21 \mathrm{TV}, 24 \mathrm{TV}, 25 \mathrm{~V}, 29 \mathrm{~T}$, 32TV, 35TV

$8 \mathrm{~T}, 12 \mathrm{~T}, 13 \mathrm{~T}, 20 \mathrm{C}, 21 \mathrm{~T}, 29 \mathrm{~T}$, $31 \mathrm{C}$

$10 \mathrm{C}$

$27 \mathrm{~T}$

Pocillopora eydouxi

Pocillopora verrucosa

Seriatopora caliendrum

Stylophora pistillata

Family Poritidae

Goniopora columna

Goniopora djiboutiensis

Goniopora lobata

Goniopora pandoraensis

Goniopora stutchburyi

Goniopora tenuidens

Porites cf. aranetai

Porites cylindrica

Porites heronensis

Porites lichen

Porites lobata

Porites lutea

Porites murrayensis

Porites rus

Porites solida

Family Siderastreidae

Pseudosiderastrea tayami

Family Thamnasteriidae

Psammocora contigua

Psammocora nierstraszi

Psammocora superficialis

Family Trachyphylliidae

Trachyphyllia geoffroyi

1C, $4 \mathrm{~T}, 12 \mathrm{TV}, 13 \mathrm{TV}, 14 \mathrm{C}$, $18 \mathrm{TV}, 20 \mathrm{~V}, 24 \mathrm{~T}, 25 \mathrm{~V}, 27 \mathrm{TV}$, 28C, 29TV, 31V, 33V, 35TV $1 \mathrm{TV}, 3 \mathrm{TV}, 8 \mathrm{~T}, 10 \mathrm{C}, 20 \mathrm{~V}$, 21TV, 35TV

$3 \mathrm{TV}, 12 \mathrm{~V}, 18 \mathrm{~T}, 20 \mathrm{C}, 21 \mathrm{TV}$, $23 \mathrm{~V}, 24 \mathrm{TV}, 27 \mathrm{TV}, 29 \mathrm{TV}$, 33T, 35TV

$1 \mathrm{~T}, 4 \mathrm{~T}, 20 \mathrm{C}, 29 \mathrm{TV}$

$20 \mathrm{C}, 21 \mathrm{~T}, 24 \mathrm{~T}, 25 \mathrm{C}, 29 \mathrm{~T}$

$20 \mathrm{C}$

$1 \mathrm{~T}, 17 \mathrm{C}, 18 \mathrm{~T}, 24 \mathrm{~T}, 29 \mathrm{TV}$

1TV, 3TV, 4TV, 6TV, 8TV, $10 \mathrm{~V}, 12 \mathrm{~V}, 13 \mathrm{TV}, 14 \mathrm{~V}, 15 \mathrm{~T}$, $16 \mathrm{TV}, 17 \mathrm{~V}, 18 \mathrm{TV}, 20 \mathrm{~V}$, 21TV, 22TV, 24TV, 25V, 29TV, 31V

$4 \mathrm{C}, 13 \mathrm{~V}, 16 \mathrm{TV}, 21 \mathrm{C}, 22 \mathrm{TV}$

$13 \mathrm{~V}$

$29 \mathrm{C}$

13T, 29TV

$31 \mathrm{C}$

$3 \mathrm{~T}, 10 \mathrm{C}, 12 \mathrm{~T}, 13 \mathrm{~T}, 17 \mathrm{C}, 27 \mathrm{~T}$, $28 \mathrm{C}, 33 \mathrm{~T}$

$1 \mathrm{~T}, 4 \mathrm{~T}, 6 \mathrm{~T}, 11 \mathrm{C}, 13 \mathrm{~T}, 21 \mathrm{C}$. $23 \mathrm{C}, 24 \mathrm{~T}, 25 \mathrm{C}, 29 \mathrm{C}, 31 \mathrm{C}$

$13 \mathrm{~T}$

$6 \mathrm{TC}, 27 \mathrm{~T}, 33 \mathrm{~T}$

$1 \dot{\mathrm{C}}, 20 \mathrm{C}, 25 \mathrm{C}$

$19 \mathrm{~T}$

$1 \mathrm{~T}, 4 \mathrm{C}, 10 \mathrm{C}, 18 \mathrm{~T}, 20 \mathrm{C}, 25 \mathrm{~V}$, $28 \mathrm{~V}$

$13 \mathrm{~T}, 18 \mathrm{~T}, 20 \mathrm{C}, 33 \mathrm{~T}$

$1 \mathrm{~T}, 4 \mathrm{~T}$

$1 \mathrm{~T}, 14 \mathrm{C}, 16 \mathrm{~T}, 17 \mathrm{C}, 18 \mathrm{~T}, 21 \mathrm{~T}$, $25 \mathrm{C}, 26 \mathrm{~T}$

$3 \mathrm{~T}, 4 \mathrm{~T}, 5 \mathrm{C}, 6 \mathrm{~T}, 12 \mathrm{~T}, 13 \mathrm{~T}, 14 \mathrm{C}$, $18 \mathrm{~T}, 21 \mathrm{~T}, 23 \mathrm{C}, 24 \mathrm{~T}, 25 \mathrm{C}$, $27 \mathrm{~T}, 29 \mathrm{~T}, 31 \mathrm{C}, 32 \mathrm{TV}$

$16 \mathrm{~T}$

$18 \mathrm{~T}$

$1 \mathrm{C}$

C (station label lost)

$25 \mathrm{C}$

$13 \mathrm{~T}$

$16 \mathrm{C}$

$2 \mathrm{C}, 10 \mathrm{C}, 11 \mathrm{C}, 17 \mathrm{~V}, 20 \mathrm{~V}, 23 \mathrm{~V}$, 29TV, 31V 
Table 2 The total number of scleractinian species recorded at each DA1/98 station and transect.

\begin{tabular}{|c|c|c|c|c|}
\hline $\begin{array}{l}\text { DA1/98 } \\
\text { Station }\end{array}$ & $\begin{array}{c}\text { Total } \\
\text { number } \\
\text { of } \\
\text { species }\end{array}$ & $\begin{array}{l}\text { Number of } \\
\text { species } \\
\text { recorded } \\
\text { from } \\
\text { transects } \\
\text { only }\end{array}$ & $\begin{array}{l}\% \text { of corals } \\
\text { occurring } \\
\text { in the } \\
\text { transect }\end{array}$ & $\begin{array}{l}\text { Number of } \\
\text { coral } \\
\text { species } / \mathrm{m}^{2}\end{array}$ \\
\hline 1 & 31 & 27 & 87 & 1.08 \\
\hline 2 & 5 & & & \\
\hline 3 & 31 & 29 & 94 & 1.16 \\
\hline 4 & 35 & 29 & 83 & 1.16 \\
\hline 5 & 6 & & & \\
\hline 6 & 19 & 14 & 74 & 0.56 \\
\hline 7 & 0 & & & \\
\hline 8 & 22 & 21 & 96 & 0.84 \\
\hline 9 & 0 & & & \\
\hline 10 & 29 & & & \\
\hline 11 & 6 & & & \\
\hline 12 & 32 & 28 & 88 & 1.12 \\
\hline 13 & 50 & 45 & 90 & 1.80 \\
\hline 14 & 21 & & & \\
\hline 15 & 4 & 4 & 100 & 0.16 \\
\hline 16 & 20 & 14 & 70 & 0.56 \\
\hline 17 & 26 & & & \\
\hline 18 & 33 & 33 & 100 & 1.32 \\
\hline 19 & 20 & 19 & 95 & 0.76 \\
\hline 20 & 44 & & & \\
\hline 21 & 28 & 27 & 96 & 1.08 \\
\hline 22 & 9 & 9 & 100 & 0.36 \\
\hline 23 & 16 & & & \\
\hline 24 & 30 & 27 & 90 & 1.08 \\
\hline 25 & 50 & & & \\
\hline 26 & 5 & 5 & 100 & 0.20 \\
\hline 27 & 23 & 22 & 96 & 0.88 \\
\hline 28 & 8 & & & \\
\hline 29 & 38 & 36 & 95 & 1.44 \\
\hline 30 & 0 & & & \\
\hline 31 & 37 & & & \\
\hline 33 & 23 & 22 & 97 & 0.88 \\
\hline 34 & 7 & & & \\
\hline 35 & 17 & 15 & 88 & 0.60 \\
\hline
\end{tabular}

Table 3 List of scleractinian coral species recorded only from intertidal DA1/98 stations.

\begin{tabular}{ll}
\hline Species & DA1/98 Station \\
\hline Acanthastrea hillae & 10 \\
Acropora anthocercis & 23 \\
Acropora aspera & 31 \\
Acropora dendrum & 17 \\
Acropora gemmifera & 14 \\
Acropora sarmentosa & 25,34 \\
Astreopora explanata & 20 \\
Catalaphyllia jardinei & 31 \\
Euphyllia divisa & 10 \\
Euphyllia glabrescens & $11,17,25,28,31,34$ \\
Favia lizardensis & 10 \\
Favia speciosa & 17 \\
Favia veroni & 17 \\
Favites complanata & 17 \\
Favites russelli & 25 \\
Fungia cyclolites & 31 \\
Goniopora columna & 31 \\
Hydnophora pilosa & 10,20 \\
Leptastrea purpurea & 10 \\
Leptastrea transversa & 5,9 \\
Lobophyllia corymbosa & $20,25,31$ \\
Montipora foveolata & 31 \\
Montipora spongodes & 17,31 \\
Montipora turgescens & $20,25,31$ \\
Montipora venosa & 17 \\
Pectinia lactuca & 20 \\
Platygyra lamellina & 20,31 \\
Platygyra ryukyuensis & $10,20,25$ \\
Platygyra verweyi & 5 \\
Psammocora contigua & 25 \\
\hline
\end{tabular}


Table 4 List of scleractinian coral species recorded only from subtidal DA1/98 stations $<10 \mathrm{~m}$ depth.

\begin{tabular}{ll}
\hline Species & DA1/98 Station \\
\hline Acropora danai & 22 \\
Acropora microphthalma & 29 \\
Acropora polystoma & 12 \\
Acropora pulchra & 8 \\
Acropora subulata & 13 \\
Acropora yongei & 8 \\
Astreopora gracilis & 1 \\
Duncanopsammia axifuga & 13 \\
Euphyllia ancora & 33 \\
Favia rotumana & 6,8 \\
Favia stelligera & 3 \\
Fungia scutaria & 13 \\
Goniastrea edwardsi & 8 \\
Goniopora pandoraensis & 13 \\
Hydnophora rigida & 24,29 \\
Montipora danae & $8,12,13$ \\
Montipora grisea & 29 \\
Montipora informis & 3,6 \\
Montipora peltiformis & 13 \\
Montipora spumosa & 29 \\
Montipora undata & 13 \\
Pocillopora verrucosa & 13 \\
Porites cf. aranetai & 19 \\
Porites murrayensis & 16 \\
Porites solida & 1 \\
Psammocora nierstraszi & 13 \\
Psammocora superficialis & 16 \\
Seriatopora caliendrum & 29 \\
Stylophora pistillata & 13,29 \\
Symphyllia agaricia & 13 \\
\hline
\end{tabular}

Table 5 List of scleractinian coral species recorded only from subtidal DA1/98 stations $>10 \mathrm{~m}$ depth.

\begin{tabular}{ll}
\hline Species & DA1/98 Station \\
\hline Acropora acuminata & 18 \\
Acropora valenciennesi & 18 \\
Australomussa rowleyensis & 27 \\
Echinopora horrida & 4 \\
Montipora crassituberculata & 21 \\
Montipora turtlensis & 4 \\
Montipora verrucosa & 4 \\
Pachyseris rugosa & 21 \\
Pavona clavus & 4 \\
Pavona varians & 4 \\
Porites rus & 18 \\
Tubastraea diaphana & 15,26 \\
\hline
\end{tabular}


Appendix 1 List of scleractinian coral species recorded from intertidal DA1/98 stations. C - sample was collected from an area other than from a transect; $\mathrm{V}$ - visual record only; $\mathrm{TrV}$ - specimen was observed on a transect; $\operatorname{TrC}$ - specimen was collected from a transect.

\begin{tabular}{|c|c|c|c|c|c|c|c|c|c|c|c|c|c|c|c|}
\hline \multirow[t]{2}{*}{ Genus } & \multirow{2}{*}{ Species } & \multicolumn{14}{|c|}{ DA1/98 Stations } \\
\hline & & 2 & 5 & 7 & 10 & 11 & 14 & 17 & 20 & 23 & 25 & 28 & 31 & 34 & 35 \\
\hline Acanthastrea & echinata & & & & & & & & C & & & & $\mathrm{C}$ & & \\
\hline Acanthastrea & hillae & & & & $\mathrm{C}$ & & & & & & & & & & \\
\hline Acropora & anthocercis & & & & & & & & & $\mathrm{C}$ & & & & & \\
\hline Acropora & aspera & & & & & & & & & & & & $\mathrm{C}$ & & \\
\hline Acropora & austera & & & & & & & $\mathrm{C}$ & & & $\mathrm{C}$ & & & & \\
\hline Acropora & clathrata & & & & & & & & & & & & & & \\
\hline Acropora & cytherea & & & & & & & & & & $\mathrm{C}$ & & & & \\
\hline Acropora & dendrum & & & & & & & $\mathrm{C}$ & & & & & & & \\
\hline Acropora & digitifera & & & & $\mathrm{C}$ & & $\mathrm{C}$ & & C & $\mathrm{C}$ & $\mathrm{C}$ & & $\mathrm{C}$ & & \\
\hline Acropora & divaricata & & & & & & & C & & & & & & & \\
\hline Acropora & formosa & & & & & & & $\mathrm{C}$ & & & & & & $\mathrm{C}$ & \\
\hline Acropora & gemmifera & & & & & & $\mathrm{C}$ & & & & & & & & \\
\hline Acropora & humilis & & & & C & & & & & & $\mathrm{C}$ & & $\mathrm{C}$ & & \\
\hline Acropora & hyacinthus & & $\mathrm{C}$ & & & & & & & C & $\mathrm{C}$ & & $\mathrm{C}$ & & \\
\hline Acropora & nobilis & & & & & & & & & & $\mathrm{C}$ & & & & \\
\hline Acropora & latistella & & & & & & & & & $\mathrm{C}$ & $\mathrm{C}$ & & & & \\
\hline Acropora & millepora & & & & C & & & & & & & & $\mathrm{C}$ & & \\
\hline Acropora & nasuta & & & & & & & & & & $\mathrm{C}$ & & & & \\
\hline Acropora & samoensis & & & & & & & $\mathrm{C}$ & & & & & $\mathrm{C}$ & & \\
\hline Acropora & sarmentosa & & & & & & & & & & $\mathrm{C}$ & & & $\mathrm{C}$ & \\
\hline Acropora & spicifera & & & & & & & & & & $\bar{C}$ & & & & \\
\hline Acropora & tenuis & & & & & & $\mathrm{C}$ & $\mathrm{C}$ & & & & & & & $\operatorname{TrC}$ \\
\hline Acropora & valida & & & & & & & & $\mathrm{C}$ & & $\mathrm{C}$ & . & C & & \\
\hline Acropora & vaughani & & & & & & & & & & & & $\mathrm{C}$ & & \\
\hline Acropora & verweyi & & & & & & & & & & & & & & $\mathrm{C}$ \\
\hline Astreopora & explanata & & & & & & & & C & & & & & & \\
\hline Astreopora & myriophthalma & & & & & & & & & & $\mathrm{V}$ & & & & \\
\hline Barabattoia & amicorum & C & & & & & & & & & $\mathrm{C}$ & & & & \\
\hline Catalaphyllia & jardinei & & & & & & & & & & & & $\mathrm{C}$ & & \\
\hline Caulastrea & tumida & & & & C & & V & V & $\mathrm{V}$ & $\mathrm{V}$ & $\mathrm{V}$ & $\mathrm{V}$ & $\mathrm{V}$ & $\mathrm{V}$ & $\operatorname{TrV}$ \\
\hline Cyphastrea & microphthalma & & & & & & & & C & & & & & & $\operatorname{TrC}$ \\
\hline Cyphastrea & serailia & & & & & & $C$ & & $\mathrm{C}$ & & $\mathrm{C}$ & & $\mathrm{C}$ & & \\
\hline Echinophyllia & aspera & & & & & & & & $\mathrm{C}$ & $\mathrm{V}$ & & & & & $\operatorname{TrV}$ \\
\hline Echinopora & lamellosa & & & & & & & & $C$ & & & & & & \\
\hline Euphyllia & divisa & & & & C & & & & & & & & & & \\
\hline Euphyllia & glabrescens & & & & & $\mathrm{C}$ & & V & & & $\mathrm{V}$ & $\mathrm{V}$ & $\mathrm{V}$ & $\mathrm{V}$ & \\
\hline Favia & favus & & & & & & $\mathrm{V}$ & & $\mathrm{C}$ & C & $\mathrm{V}$ & & $\mathrm{V}$ & & $\operatorname{TrV}$ \\
\hline Favia & lizardensis & & & & C & & & & & & & & & & \\
\hline Favia & matthaii & & & & & & & & & & $\mathrm{C}$ & & & & \\
\hline Favia & pallida & & & & & & $\mathrm{C}$ & & C & $\mathrm{C}$ & $\mathrm{C}$ & & C & & \\
\hline Favia & rotundata & & & & $\mathrm{C}$ & C & & $\mathrm{C}$ & & & & $\mathrm{C}$ & $\mathrm{C}$ & & \\
\hline Favia & speciosa & & & & & & & $\mathrm{C}$ & & & & & & & \\
\hline Favia & veroni & & & & & & & C & & & & & & & \\
\hline Favites & abdita & & & & & & $\mathrm{V}$ & & $\mathrm{C}$ & & & & & $\mathrm{V}$ & $\operatorname{TrV}$ \\
\hline Favites & chinensis & C & & & & & & & & & & & & & \\
\hline Favites & complanata & & & & & & & $\mathrm{C}$ & & & & & & & \\
\hline Favites & flexuosa & & & & $\mathrm{C}$ & & $C$ & & & & C & & $\mathrm{C}$ & & \\
\hline Favites & halicora & C & & & & & & & & & $\mathrm{C}$ & & & & \\
\hline Favites & pentagona & & & & $\mathrm{C}$ & & & & & & & & & & \\
\hline Favites & russelli & & & & & & & & & & $\mathrm{C}$ & & & & \\
\hline Fungia & cyclolites & & & & & & & & & & & & $\mathrm{C}$ & & \\
\hline Fungia & fungites & & & & C & & $\mathrm{C}$ & C & $\mathrm{C}$ & & $\mathrm{C}$ & & C & & $\mathrm{C}, \operatorname{TrC}$ \\
\hline Galaxea & astreata & & & & & & $\mathrm{C}$ & & $\mathrm{V}$ & & $\mathrm{V}$ & C & V & & $\mathrm{TrV}$ \\
\hline Galaxea & fascicularis & & & & C & & & & $\mathrm{V}$ & & & & & & $\mathrm{TrV}$ \\
\hline Goniastrea & aspera & C & $\mathrm{V}$ & & C & C & & $\mathrm{C}$ & C & $\mathrm{C}$ & & $c$ & $\mathrm{C}$ & & \\
\hline Goniastrea & australensis & & & & & & $\mathrm{V}$ & & & & & & & & \\
\hline Goniastrea & favulus & & & & & & & & $\mathrm{C}$ & & & & & & \\
\hline Goniastrea & palauensis & & & & & & & & & & C & & & & \\
\hline Goniastrea & retiformis & & & & & & & & C & & $\mathrm{C}$ & & & & $\operatorname{TrV}$ \\
\hline Goniopora & columna & & & & & & & & & & & & $\mathrm{C}$ & & \\
\hline
\end{tabular}


Appendix 1 (cont.)

\begin{tabular}{|c|c|c|c|c|c|c|c|c|c|c|c|c|c|c|c|}
\hline \multirow[t]{2}{*}{ Genus } & \multirow[t]{2}{*}{ Species } & \multicolumn{14}{|c|}{ DA1/98 Stations } \\
\hline & & 2 & 5 & 7 & 10 & 11 & 14 & 17 & 20 & 23 & 25 & 28 & 31 & 34 & 35 \\
\hline Goniopora & djiboutiensis & & & & $\mathrm{C}$ & & & C & & & & $\mathrm{C}$ & & & \\
\hline Goniopora & lobata & & & & & $\mathrm{C}$ & & & & $\mathrm{C}$ & $\mathrm{C}$ & & C & & \\
\hline Goniopora & tenutidens & & & & & & & & $\mathrm{C}$ & & $\mathrm{C}$ & & & & \\
\hline Herpolitha & $\operatorname{limax}$ & & & & $\mathrm{V}$ & & & C & C & & $\mathrm{V}$ & & & & \\
\hline Hydnophora & exesa & & & & & & & & & & $\mathrm{C}$ & & & & \\
\hline Hydnophora & microconos & & & & & & C & & & & & & & & \\
\hline Hydnophora & pilosa & & & & $\mathrm{C}$ & & & & $\mathrm{C}$ & & & & & & \\
\hline Leptastrea & purpurea & & & & $\mathrm{C}$ & & & & & & & & & & \\
\hline Leptastrea & transversa & & $\mathrm{C}$ & & $\mathrm{C}$ & & & & & & & & & & \\
\hline Leptoria & phrygia & & & & & & & & & & V & & & & $\operatorname{TrV}$ \\
\hline Lobophyllia & corymbosa & & & & & & & & C & & $\mathrm{V}$ & & $\mathrm{V}$ & & \\
\hline Lobophyllia & hemprichii & & & & $\mathrm{C}$ & & $\mathrm{V}$ & $\mathrm{V}$ & $\mathrm{C}$ & $\mathrm{C}$ & $\mathrm{V}$ & & C & & $\operatorname{TrV}$ \\
\hline Merulina & ampliata & & & & & & & V & C & & $\mathrm{V}$ & & & & $\operatorname{TrV}$ \\
\hline Montastrea & magnistellata & & & & & & & & $\mathrm{C}$ & & & & & & \\
\hline Montastrea & valenciennesi & & & & $C$ & & & & C & & & & & & \\
\hline Montipora & aequituberculata & & & & & & & & & & & & & $\mathrm{C}$ & \\
\hline Montipora & caliculata & & & & & & & & & & & $\mathrm{C}$ & & & \\
\hline Montipora & capricornis & & & & & & & & & & $\mathrm{C}$ & & & & \\
\hline Montipora & efflorescens & & & & & & & & $\mathrm{C}$ & & & & & & \\
\hline Montipora & foveolata & & & & & & & & & & & & C & & \\
\hline Montipora & hispida & & & & & & & & C & & & & & & \\
\hline Montipora & mollis & & & & & & & & & & $\mathrm{C}$ & & & $C$ & \\
\hline Montipora & spongodes & & & & & & & $\mathrm{C}$ & & & & & $\mathrm{C}$ & & \\
\hline Montipora & tuberculosa & & & & & & & & & & & & C & & \\
\hline Montipora & turgescens & & & & & & & & C & & $C$ & & $\mathrm{C}$ & & \\
\hline Montipora & venosa & & & & & & & C & & & & & & & \\
\hline Moseleya & latistellata & & & & C & & & & & & $\mathrm{C}$ & & C & & \\
\hline Mycedium & elephantotus & & & & & & & & $\mathrm{C}$ & & & & & & \\
\hline Oulophyllia & bennettae & & & & & & & & $C$ & & & & & & \\
\hline Oxypora & lacera & & & & & & & & C & & $C$ & & & & \\
\hline Pavona & decussata & & & & & & & & $\mathrm{V}$ & & $\mathrm{V}$ & & & & $\mathrm{C}$ \\
\hline Pectinia & lactuca & & & & & & & & $\mathrm{C}$ & & & & & & \\
\hline Pectinia & paeonia & & & & & & & C & & & & & & & \\
\hline Platygyra & daedalea & & & & $C$ & & & & C & $\mathrm{C}$ & $C$ & & & & \\
\hline Platyoyra & lamellina & & & & & & & & C & & & & $\mathrm{C}$ & & \\
\hline Platygyra & ryukyulensis & & & & $C$ & & & & $\mathrm{C}$ & & $C$ & & & & \\
\hline Platygyra & sinensis & & C & & $C$ & & $C$ & & & & $C$ & & & & \\
\hline Platygyra & verweyi & & $\mathrm{C}$ & & & & & & & & & & & & \\
\hline Plesiastrea & versipora & & & & & & $\mathrm{C}$ & & & $\mathrm{V}$ & $\mathrm{V}$ & & $\mathrm{V}$ & & $\operatorname{TrV}$ \\
\hline Pocillopora & damicornis & & & & $\mathrm{V}$ & & V & $\mathrm{V}$ & $\mathrm{V}$ & & $\mathrm{V}$ & & V & & \\
\hline Podabacia & crustacea & & & & & & & & C & & & & & & \\
\hline Porites & cylindrica & & & & $C$ & & & & $\mathrm{C}$ & & $\mathrm{V}$ & $\mathrm{V}$ & & & \\
\hline Porites & heronensis & & & & & & & & $\mathrm{C}$ & & & & & & \\
\hline Porites & lobata & & & & & & $\mathrm{C}$ & $\mathrm{C}$ & & & $\mathrm{C}$ & & & & \\
\hline Porites & lutea & & $\mathrm{C}$ & & & & $\mathrm{C}$ & & & $\mathrm{C}$ & $\mathrm{C}$ & & C & & \\
\hline Psammocora & contigua & & & & & & & & & & $\mathrm{C}$ & & & & \\
\hline Stylocoeniella & guentheri & & & & & & & & C & & & & & & \\
\hline Trachyphyllia & geoffroyi & C & & & $\mathrm{C}$ & $\mathrm{C}$ & & V & $\mathrm{V}$ & $\mathrm{V}$ & & & $\mathrm{V}$ & & \\
\hline Turbinaria & bifrons & & & & $C$ & $\mathrm{C}$ & C & V & & & $\mathrm{C}$ & & & & \\
\hline Turbinaria & conspicua & & & & & & C & & & & & & & & \\
\hline Turbinaria & peltata & & & & $\mathrm{C}$ & & & & & & & & $\mathrm{C}$ & & \\
\hline Turbinaria & reniformis & & & & & & & V & C & $\mathrm{C}$ & & & $\mathrm{V}$ & & $\operatorname{TrV}$ \\
\hline
\end{tabular}


Appendix 2 List of scleractinian coral species recorded from subtidal DA1/98 stations at depths $<10 \mathrm{~m}$. C - sample was collected from an area other than from a transect; $\mathrm{V}$ - visual record only; $\operatorname{TrV}$ - specimen was observed on a transect; $\operatorname{TrC}$ - specimen was collected from a transect.

\begin{tabular}{|c|c|c|c|c|c|c|c|c|c|c|c|c|c|c|}
\hline \multirow[t]{2}{*}{ Genus } & \multirow[t]{2}{*}{ Species } & \multicolumn{13}{|c|}{ DA1/98 Stations } \\
\hline & & 1 & 3 & 6 & 8 & 9 & 12 & 13 & 16 & 19 & 22 & 24 & 29 & 33 \\
\hline Acanthastrea & echinata & & & & $\operatorname{TrC}$ & & $\operatorname{TrC}$ & $\operatorname{Tr} C$ & & & & & $\operatorname{TrC}$ & \\
\hline Acropora & aculeus & $\operatorname{TrC}$ & & & & & & $\operatorname{TrC}$ & & & & & & \\
\hline Acropora & austera & & & & & & & & & & $\operatorname{TrC}$ & & & \\
\hline Acropora & cerealis & & & & & & & $\operatorname{TrC}$ & & & & & & \\
\hline Acropora & clathrata & & & & & & & $\operatorname{TrC}$ & & & $\operatorname{TrC}$ & & & \\
\hline Acropora & cytherea & & 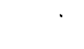 & & $\operatorname{TrC}$ & & $\operatorname{TrC}$ & $\operatorname{TrC}$ & $\mathrm{C}$ & $\operatorname{TrC}$ & $\operatorname{TrC}$ & & & \\
\hline Acropora & danai & & & & & & & & & & $\operatorname{TrC}$ & & & \\
\hline Acropora & digitifera & & $\mathrm{C}$ & $\mathrm{C}$ & & & & & & $\operatorname{TrC}$ & & & & \\
\hline Acropora & divaricata & & & & $\operatorname{TrC}$ & & & & & & & & & \\
\hline Acropora & florida & $\operatorname{TrV}$ & & & & & $\operatorname{TrC}$ & $\mathrm{V}$ & & & & $\operatorname{TrC}$ & & \\
\hline Acropora & formosa & & & & & & & $\operatorname{TrC}$ & & $\operatorname{TrC}$ & & $\mathrm{C}$ & & \\
\hline Acropora & humilis & & & & & & & & $\operatorname{TrC}$ & & & & & \\
\hline Acropora & hyacinthus & & $\operatorname{TrC}$ & & $\operatorname{TrC}$ & & & & $\operatorname{TrC}$ & & & & & \\
\hline Acropora & nobilis & & $\operatorname{TrC}$ & & & & $\operatorname{TrC}$ & $\operatorname{TrC}$ & & & $\operatorname{TrC}$ & & $\operatorname{TrC}$ & \\
\hline Acropora & latistella & & & & & & & & $\mathrm{C}$ & & & $\operatorname{TrC}$ & & \\
\hline Acropora & microphthalma & & & & & & & & & & & & $\operatorname{TrC}$ & \\
\hline Acropora & millepora & & & & & & & & & & & & $\operatorname{TrC}$ & \\
\hline Acropora & nasuta & & $\operatorname{TrC}$ & $\operatorname{TrC}$ & & & $\operatorname{TrC}$ & & & $\operatorname{TrC}$ & $\operatorname{TrC}$ & $\operatorname{TrC}$ & $\operatorname{TrC}$ & \\
\hline Acropora & polystoma & & & & & & $\operatorname{TrC}$ & & & & & & & \\
\hline Acropora & pulchra & & & & $\operatorname{TrC}$ & & & & & & & & & \\
\hline Acropora & samoensis & & $\operatorname{TrC}$ & & & & & & & & & $\operatorname{TrC}$ & $\operatorname{TrC}$ & \\
\hline Acropora & secale & & & & & & & & & & & $\operatorname{TrC}$ & $\operatorname{TrC}$ & \\
\hline Acropora & selago & $\operatorname{TrC}$ & $\operatorname{TrC}$ & & & & & & & & & & & \\
\hline Acropora & solitaryensis & & & & $\operatorname{TrC}$ & & & & & & & & & \\
\hline Acropora & spicifera & & & & $\operatorname{TrC}$ & & & $\operatorname{TrC}$ & & & & & & \\
\hline Acropora & subulata & & & & & & & $\operatorname{TrC}$ & & & & & & \\
\hline Acropora & tenuis & & & & & & & & & $\operatorname{TrC}$ & & $\operatorname{TrC}$ & $\operatorname{TrC}$ & \\
\hline Acropora & valida & & & & & & & & & $\operatorname{TrC}$ & & $\operatorname{TrC}$ & $\operatorname{TrC}$ & \\
\hline Acropora & verweyi & & & & & & & & & & & $\mathrm{C}$ & & \\
\hline Acropora & yongei & & & & $\operatorname{TrC}$ & & & & & & & & & \\
\hline Astreopora & gracilis & $\operatorname{TrC}$ & & & & & & & & & & & & \\
\hline Astreopora & myriophthalma & & & & & & $\operatorname{TrC}$ & $\operatorname{TrV}$ & & & & $\operatorname{TrC}$ & & $\operatorname{TrC}$ \\
\hline Barabattoia & amicorum & $\operatorname{TrC}$ & & & & & & & & & & $\operatorname{TrC}$ & & $\operatorname{TrC}$ \\
\hline Caulastrea & tumida & $\operatorname{TrC}$ & & & & & & & & & & $\operatorname{TrV}$ & $\operatorname{TrV}$ & . \\
\hline Cyphastrea & microphthalma & $\operatorname{TrC}$ & $\operatorname{TrC}$ & & & & $\operatorname{TrC}$ & & & $\operatorname{TrV}$ & & $\operatorname{TrV}$ & & \\
\hline Cyphastrea & serailia & & & $\operatorname{TrC}$ & & & & & $\operatorname{TrC}$ & & & & & $\operatorname{TrC}$ \\
\hline Duncanopsammia & axifuga & & & & & & & $\mathrm{C}$ & & & & & & \\
\hline Echinophyllia & aspera & & $\operatorname{TrV}$ & & & & V & & & & & $\operatorname{Tr} \mathrm{V}$ & $\operatorname{TrV}$ & $\operatorname{TrC}$ \\
\hline Echinopora & lamellosa & & & & & & & & & & & & $\operatorname{TrC}$ & \\
\hline Euphyllia & ancora & & & & & & & & & & & & & $\operatorname{TrC}$ \\
\hline Favia & favus & & & & $\operatorname{TrC}$ & & $\operatorname{Tr} C$ & & & & & & $\operatorname{TrV}$ & $\operatorname{TrV}$ \\
\hline Favia & matthaii & & & & & & & & & $\operatorname{TrC}$ & & & & \\
\hline Favia & pallida & & $\operatorname{TrC}$ & & & & $\operatorname{TrC}$ & $\operatorname{TrC}$ & $\mathrm{C}$ & $\operatorname{TrC}$ & & & & \\
\hline Eavia & rotumana & & & $\mathrm{C}$ & $\operatorname{TrC}$ & & & & & & & & & \\
\hline Favia & rotundata & $\operatorname{Tr} C$ & & & & & & & & & & & & \\
\hline Favia & stelligera & & $\operatorname{TrC}$ & & & & & & & & & & & \\
\hline Favites & $a b d i t a$ & $\operatorname{Tr} C$ & $\operatorname{TrC}$ & & & & $\operatorname{TrC}$ & & & & & $\operatorname{TrC}$ & $\operatorname{TrV}$ & $\operatorname{TrV}$ \\
\hline Favites & chinensis & & & & $\operatorname{TrC}$ & & & & $\operatorname{TrC}$ & & & & & \\
\hline Favites & flexuosa & & & & & & $\operatorname{TrC}$ & $\operatorname{TrC}$ & & & & & & \\
\hline Favites & halicora & & & & & & & $\operatorname{TrC}$ & & & & & & \\
\hline Favites & pentagona & & & & & & & & $\operatorname{TrC}$ & $\operatorname{TrC}$ & & & & \\
\hline Fungia & fungites & $\operatorname{TrC}$ & & & & & C & C,TrC & & & & V & $\operatorname{TrV}$ & \\
\hline Fungia & scutaria & & & & & & & C & & & & & & \\
\hline Galaxea & astreata & C & & & & & $\operatorname{TrV}$ & $\operatorname{TrV}$ & & & & $\operatorname{TrC}$ & $\operatorname{TrV}$ & V \\
\hline Galaxea & fascicularis & $\operatorname{TrV}$ & $\operatorname{TrV}$ & & $\operatorname{TrC}$ & & & & & & & & & \\
\hline Goniastrea & aspera & & & $\operatorname{TrC}, \mathrm{C}$ & $\operatorname{TrC}$ & & $\operatorname{TrC}$ & $\operatorname{TrC}$ & & & & & & \\
\hline Goniastrea & australensis & & & & & & & & & & & $\operatorname{TrV}$ & & \\
\hline Goniastrea & edwardsi & & & & $\operatorname{Tr} C$ & & & & & & & & & \\
\hline Goniastrea & favulus & & $\operatorname{TrC}$ & & & & & & & & & & & \\
\hline Goniastrea & palauensis & & & & & & & & $\operatorname{TrC}$ & & & & & \\
\hline
\end{tabular}


Appendix 2 (cont.)

\begin{tabular}{|c|c|c|c|c|c|c|c|c|c|c|c|c|c|c|}
\hline \multirow[t]{2}{*}{ Genus } & \multirow[t]{2}{*}{ Species } & \multicolumn{13}{|c|}{ DA1/98 Stations } \\
\hline & & 1 & 3 & 6 & 8 & 9 & 12 & 13 & 16 & 19 & 22 & 24 & 29 & 33 \\
\hline Goniastrea & pectinata & $\operatorname{TrC}, \mathrm{C}$ & & & & & & $\operatorname{TrC}$ & & C & & & & \\
\hline Goniastrea & retiformis & & $\operatorname{TrC}$ & & $\operatorname{TrC}$ & & $\operatorname{TrC}$ & & & & & & & \\
\hline Goniopora & djiboutiensis & & $\operatorname{TrC}$ & & & & $\operatorname{TrC}$ & $\operatorname{TrC}$ & & & & & & $\operatorname{TrC}$ \\
\hline Goniopora & lobata & $\operatorname{TrC}$ & & $\operatorname{TrC}$ & & & & $\operatorname{TrC}$ & & & & $\operatorname{TrC}$ & C & \\
\hline Goniopora & pandoraensis & & & & & & & $\operatorname{TrC}$ & & & & & & \\
\hline Goniopora & stutchburyi & & & $\operatorname{TrC}, \mathrm{C}$ & & & & & & & & & & $\operatorname{TrC}$ \\
\hline Goniopora & tenuidens & C & & & & & & & . & & & & . & \\
\hline Herpolitha & $\operatorname{limax}$ & $\operatorname{TrC}$ & & & & & & $\operatorname{TrC}$ & & $\operatorname{Tr} C$ & & $\operatorname{TrC}$ & $\operatorname{TrV}$ & $\operatorname{TrV}$ \\
\hline Hydnophora & exesa & & & & & & & $\operatorname{TrC}$ & & $\operatorname{TrV}$ & & & & $\operatorname{TrV}$ \\
\hline Hydnophora & microconos & & & & & & & $\operatorname{TrC}$ & & & & & & \\
\hline Hydnophora & rigida & & & & & & & & & & & $\operatorname{TrC}$ & $\operatorname{TrV}$ & \\
\hline Leptoria & phrygia & & & & & & $\operatorname{TrC}$ & & & & & & & $\operatorname{TrV}$ \\
\hline Lobophyllia & hataii & & & & & & & $\operatorname{TrC}$ & & & & & & \\
\hline Lobophyllia & hemprichii & $\operatorname{TrC}$ & $\operatorname{TrV}$ & & & & $\operatorname{TrV}$ & $\operatorname{TrV}$ & & & $\operatorname{TrV}$ & $\operatorname{TrV}$ & $\operatorname{TrV}$ & $\operatorname{TrV}$ \\
\hline Merulina & ampliata & & $\operatorname{TrV}$ & & & & $\operatorname{TrV}$ & $\operatorname{TrV}$ & & & & $\operatorname{TrV}$ & $\operatorname{Tr} C$ & \\
\hline Montastrea & curta & & $\operatorname{TrC}$ & C & & & C & & & & & & & \\
\hline Montastrea & magnistellata & & & & & & & $\operatorname{TrC}$ & & $\operatorname{TrC}$ & & & $\operatorname{TrV}$ & \\
\hline Montastrea & valenciennesi & $\operatorname{TrC}$ & & & & & & & & & & & & \\
\hline Montipora & aequituberculata & & & & & & & & & $\operatorname{TrC}$ & & & $\operatorname{TrC}$ & \\
\hline Montipora & caliculata & & & & & & & & & $\operatorname{TrC}$ & & & & \\
\hline Montipora & capricornis & & & $\operatorname{TrC}$ & & & & & & & & & & \\
\hline Montipora & danae & & & & $\operatorname{TrC}$ & & $\operatorname{TrC}$ & $\operatorname{TrC}$ & & & & & & \\
\hline Montipora & efflorescens & & & & & & & & & & & & $\operatorname{TrC}$ & \\
\hline Montipora & foliosa & & & & & & & & & & & & $\operatorname{TrC}$ & \\
\hline Montipora & grisea & & & & & & & & & & & & $\operatorname{Tr} C$ & \\
\hline Montipora & hispida & & $\operatorname{TrC}$ & & & & & $\operatorname{TrC}$ & & $\operatorname{TrC}$ & & & $\operatorname{TrC}$ & $\operatorname{TrC}$ \\
\hline Montipora & hoffmeisteri & & & & & & & $\operatorname{TrC}$ & & $\operatorname{TrC}$ & & & $\operatorname{TrC}$ & \\
\hline Montipora & informis & & $\operatorname{TrC}$ & $\mathrm{C}$ & & & & & & & & & & \\
\hline Montipora & millepora & $\operatorname{TrC}$ & & & & & & & & & & & & \\
\hline Montipora & mollis & & & $\operatorname{TrC}$ & & & & $\operatorname{TrC}$ & & & & & & $\operatorname{TrC}$ \\
\hline Montipora & monasteriata & & $\operatorname{TrC}$ & & & & & $\operatorname{TrC}$ & & & & & & $\operatorname{TrC}$ \\
\hline Montipora & peltiformis & & & & & & & $\operatorname{TrC}$ & & & & & & \\
\hline Montipora & spumosa & & & & & & & & & & & & $\operatorname{TrC}$ & \\
\hline Montipora & stellata & $\operatorname{TrC}$ & & & & & & & & & & & & \\
\hline Montipora & unndata & & & & & & & $\operatorname{TrC}$ & & & & & & \\
\hline Moseleya & latistellata & & & & & & & & & & & & & $\operatorname{TrV}$ \\
\hline Mycedium & elephantotus & $\operatorname{TrC}$ & & & & & & & & & & & $\operatorname{TrV}$ & \\
\hline Oulophyllia & bennettae & & & & & & & & $\operatorname{TrC}$ & & & & & \\
\hline Oxypora & lacera & & & & & & & & & & & $\operatorname{TrC}$ & $\operatorname{TrC}$ & \\
\hline Pachyseris & speciosa & & $\operatorname{TrC}$ & & & & & & & & & & & \\
\hline Pavona & decussata & & $\operatorname{TrC}$ & & & & $\operatorname{TrV}$ & $\operatorname{TrV}$ & & & & $\operatorname{TrV}$ & $\operatorname{Tr} \mathrm{C}$ & \\
\hline Pectinia & paeonia & $\operatorname{TrC}$ & & & & & & & & & & $\mathrm{TrC}$ & TrV & \\
\hline Platygyra & daedalea & & $\operatorname{TrC}$ & $\mathrm{C}$ & $\operatorname{TrC}$ & & $\operatorname{TrC}$ & $\operatorname{TrC}$ & $\operatorname{TrC}$ & & & & & $\operatorname{TrV}$ \\
\hline Platygyra & pini & $\operatorname{TrC}$ & & & $\operatorname{TrC}$ & & & & & & & & & \\
\hline Plesiastrea & versipora & & & $\operatorname{TrC}$ & & & $\operatorname{TrC}$ & & C & & & & & \\
\hline Pocillopora & damicornis & $\operatorname{TrV}$ & $\operatorname{TrV}$ & $\operatorname{TrV}$ & $\operatorname{TrV}$ & & $\mathrm{V}$ & $\operatorname{TrV}$ & $\operatorname{TrV}$ & & $\operatorname{TrV}$ & $\operatorname{TrV}$ & $\operatorname{TrV}$ & \\
\hline Pocillopora & eydouxi & & & & & & & $\mathrm{V}$ & $\operatorname{TrV}$ & & $\operatorname{TrV}$ & & & \\
\hline Pocillopora & verrucosa & & & & & & & $\mathrm{V}$ & & & & & & \\
\hline Podabacia & crustacea & & $\operatorname{TrC}$ & & & & $\operatorname{TrC}$ & & & & & & & \\
\hline Polyphyllia & talpina & & & & & & & $\operatorname{TrV}$ & & & & & & \\
\hline Porites & cf. aranetai & & & & & & & & & $\operatorname{Tr} C$ & & & & \\
\hline Porites & cylindrica & $\operatorname{TrC}$ & & & & & & & & & & & & \\
\hline Porites & heronensis & & & & & & & $\operatorname{TrC}$ & & & & & & $\operatorname{TrC}$ \\
\hline Porites & lichen & $\operatorname{TrC}$ & & & & & & & & & & & & \\
\hline Porites & lobata & $\operatorname{TrC}$ & & & & & & & $\operatorname{TrC}$ & & & & & \\
\hline Porites & lutea & & $\operatorname{TrC}$ & $\operatorname{TrC}$ & & & $\operatorname{TrC}$ & $\operatorname{TrC}$ & & & & $\operatorname{TrC}$ & $\operatorname{TrC}$ & \\
\hline Porites & murrayensis & & & & & & & & $\operatorname{TrC}$ & & & & & \\
\hline Porites & solida & C & & & & & & & & & & & & \\
\hline Psammocora & nierstraszi & & & & & & & $\operatorname{Tr} C$ & & & & & & \\
\hline Psammocora & superficialis & & & & & & & & $\mathrm{C}$ & & & & & \\
\hline Seriatopora & caliendrum & & & & & & & & . & & & & $\mathrm{C}$ & \\
\hline Stylophora & pistillata & & & & & & & $\operatorname{TrC}$ & & & & & $\operatorname{TrV}$ & \\
\hline
\end{tabular}


Appendix 2 (cont.)

\begin{tabular}{|c|c|c|c|c|c|c|c|c|c|c|c|c|c|c|}
\hline \multirow{2}{*}{ Genus } & \multirow[t]{2}{*}{ Species } & \multicolumn{13}{|c|}{ DA1/98 Stations } \\
\hline & & 1 & 3 & 6 & 8 & 9 & 12 & 13 & 16 & 19 & 22 & 24 & 29 & 33 \\
\hline Symphyllia & agaricia & & & & & & & $\operatorname{TrV}$ & & & & & & \\
\hline Symphyllia & recta & & $\operatorname{TrC}$ & & $\operatorname{TrC}$ & & & & & & & & & \\
\hline Trachyphyllia & geoffroyi & & & & & & & & & & & & $\operatorname{TrV}$ & \\
\hline Tubastraea & coccinea & & $\mathrm{C}$ & & & & & & & & & & & \\
\hline Turbinaria & bifrons & & & $\operatorname{TrC}$ & & & & & & & & & & \\
\hline Turbinaria & conspicua & & & & & & & & & & & & & $\operatorname{TrC}$ \\
\hline Turbinaria & frondens & $\mathrm{C}$ & & & $\mathrm{C}$ & & & $\operatorname{TrC}$ & & & & $\operatorname{TrC}$ & & $\operatorname{TrC}$ \\
\hline Turbinaria & mesenterina & $\operatorname{TrC}$ & & & & & & & & & & & & \\
\hline Turbinaria & peltata & & & $\operatorname{TrC}, \mathrm{C}$ & & & & $\operatorname{TrC}$ & C & & & & & $\operatorname{TrC}$ \\
\hline Turbinaria & reniformis & $\operatorname{TrC}$ & $\operatorname{TrC}$ & $\operatorname{TrC}$ & & & $\operatorname{TrC}$ & & $\mathrm{TrC}$ & & & $\operatorname{TrV}$ & & \\
\hline Turbinaria & stellulata & & & & & & $\operatorname{TrC}$ & & $\operatorname{TrC}$ & & & & & \\
\hline
\end{tabular}


Appendix 3 List of scleractinian coral species recorded from subtidal DA1/98 stations at depths $>10 \mathrm{~m}$. C - sample was collected from an area other than from a transect; $\mathrm{V}$ - visual record only; $\operatorname{Tr} \mathrm{V}$ - specimen was observed on a transect; $\mathrm{TrC}$ - specimen was collected from a transect.

\begin{tabular}{|c|c|c|c|c|c|c|c|c|c|}
\hline \multirow[t]{2}{*}{ Genus } & \multirow[t]{2}{*}{ Species } & \multicolumn{8}{|c|}{ DA1/98 Stations } \\
\hline & & 4 & 15 & 18 & 21 & 26 & 27 & 30 & 32 \\
\hline Acanthastrea & echinata & & & & $\operatorname{TrC}$ & & & & \\
\hline Acropora & aculeus & & & & $\operatorname{TrC}$ & & $\operatorname{TrC}, \mathrm{C}$ & & \\
\hline Acropora & acuminata & & & $\operatorname{TrC}$ & & & & & \\
\hline Acropora & cerealis & & & & $\operatorname{TrC}$ & & & & \\
\hline Acropora & florida & $\operatorname{TrV}$ & & & & & & & \\
\hline Acropora & nobilis & & & & & & & & $\mathrm{C}$ \\
\hline Acropora & latistella & $\operatorname{TrC}$ & & & & & & & \\
\hline Acropora & nasuta & $\mathrm{C}$ & & $\operatorname{TrC}$ & $\operatorname{TrC}$ & & & & \\
\hline Acropora & secale & & & & & & $\operatorname{TrC}$ & & \\
\hline Acropora & selago & $\operatorname{TrC}$ & & & & & & & \\
\hline Acropora & solitaryensis & $\operatorname{TrC}$ & & & & & & & \\
\hline Acropora & tenuis & & & & $\operatorname{TrC}$ & & & & \\
\hline Acropora & valenciennesi & & & $\operatorname{TrC}$ & & & & & \\
\hline Acropora & valida & & & $\mathrm{TrC}$ & & & & & \\
\hline Astreopora & myriophthalma & $\operatorname{TrC}$ & & $\operatorname{TrV}$ & & & $\operatorname{TrC}$ & & \\
\hline Australomussa & rowleyensis & & & & & & $\operatorname{TrC}$ & & \\
\hline Barabattoia & amicortum & & & $\operatorname{TrC}$ & & & $\operatorname{TrC}$ & & \\
\hline Cyphastrea & microphthalma & & & $\operatorname{TrC}$ & $\operatorname{TrC}$ & & & & \\
\hline Echinophyllia & aspera & & & $\operatorname{TrC}$ & $\operatorname{TrV}$ & & $\operatorname{TrV}$ & & \\
\hline Echinopora & horrida & $\operatorname{TrC}$ & & & & & & & \\
\hline Echinopora & lamellosa & & & $\operatorname{TrC}$ & & & & & \\
\hline Favia & favus & $\operatorname{TrC}, \mathrm{C}$ & & $\mathrm{TrV}$ & & $\operatorname{TrC}$ & & & \\
\hline Favia & matthaii & & & $\operatorname{TrC}$ & & & $\operatorname{TrC}$ & & \\
\hline Favia & rotundata & & & & $\operatorname{TrC}$ & & & & \\
\hline Favites & abdita & $\operatorname{TrC}$ & & $\operatorname{TrC}$ & $\operatorname{TrC}$ & & & & \\
\hline Favites & flexuosa & & & & $\operatorname{TrC}$ & & & & \\
\hline Favites & pentagona & $\operatorname{TrC}$ & & & & & & & \\
\hline Fungia & fungites & C & & $\operatorname{TrC}$ & $\operatorname{TrC}$ & & & & \\
\hline Galaxea & astreata & $\operatorname{TrC}$ & & $\operatorname{TrV}$ & & & $\operatorname{TrV}$ & & $\operatorname{TrV}$ \\
\hline Galaxea & fascicularis & & & & $\operatorname{TrV}$ & & & & $\operatorname{TrV}$ \\
\hline Goniastrea & favulus & & & $\operatorname{TrC}$ & $\operatorname{TrC}$ & & & & \\
\hline Goniastrea & palauensis & $\operatorname{TrC}$ & & & & & & & \\
\hline Goniastrea & pectinata & & & & $\operatorname{TrC}$ & & $\operatorname{TrC}$ & & \\
\hline Goniopora & djiboutiensis & & & & & & $\operatorname{TrC}$ & & \\
\hline Goniopora & lobata & $\operatorname{TrC}$ & & & $\operatorname{TrC}$ & & & & \\
\hline Goniopora & stutchburyi & & & & & & $\operatorname{TrC}$ & & \\
\hline Herpolitha & limax & & & & & & & & $\mathrm{C}$ \\
\hline Hydnophora & exesa & & & $\operatorname{TrC}$ & & & & & \\
\hline Lobophyllia & hataï & $\mathrm{C}$ & & & & & & & \\
\hline Lobophyllia & hemprichii & $\operatorname{TrC}$ & $\operatorname{TrV}$ & $\operatorname{TrV}$ & $\operatorname{TrV}$ & & & & \\
\hline Merulina & ampliata & & & & $\operatorname{TrV}$ & & & & $\mathrm{TrV}$ \\
\hline Montastrea & curta & $\operatorname{TrC}$ & & $\operatorname{TrC}$ & & & & & \\
\hline Montastrea & valenciennesi & $\mathrm{TrC}$ & & & & & $\operatorname{TrC}$ & & \\
\hline Montipora & crassituberculata & & & & $\operatorname{TrC}$ & & & & \\
\hline Montipora & efflorescens & & & & $\operatorname{TrC}$ & & & & \\
\hline Montipora & foliosa & & & $\operatorname{TrC}$ & & & & & \\
\hline Montipora & hispida & & & $\operatorname{TrC}$ & & $\operatorname{TrC}$ & & & \\
\hline Montipora & hoffmeisteri & & & $\operatorname{TrC}$ & & & & & \\
\hline Montipora & millepora & & & & & & $\operatorname{TrC}$ & & \\
\hline Montipora & monasteriata & & & $\operatorname{Tr} C$ & & & $\operatorname{TrC}$ & & \\
\hline Montipora & stellata & & & $\operatorname{TrC}$ & & & & & \\
\hline Montipora & tuberculosa & & & & $\operatorname{TrC}$ & & & & \\
\hline Montipora & turtlensis & $\operatorname{TrC}$ & & & & & & & \\
\hline Montipora & verrucosa & $\operatorname{TrC}$ & & & & & & & \\
\hline Moseleya & latistellata & $\operatorname{TrV}, \mathrm{C}$ & & & & & $\operatorname{TrV}$ & & \\
\hline Mycedium & elephantotus & $\operatorname{TrC}$ & & & & & & & \\
\hline Oxypora & lacera & & & & $\operatorname{TrC}$ & & & & \\
\hline Pachyseris & rugosa & & & & $\operatorname{TrC}$ & & & & \\
\hline Pachyseris & speciosa & & $\operatorname{TrC}$ & & & & $\mathrm{C}$ & & \\
\hline Pavona & clavus & $\operatorname{TrC}$ & & & & & & & \\
\hline
\end{tabular}


Appendix 3 (cont.)

\begin{tabular}{|c|c|c|c|c|c|c|c|c|c|}
\hline \multirow[t]{2}{*}{ Genus } & \multirow[t]{2}{*}{ Species } & \multicolumn{8}{|c|}{ DA1/98 Stations } \\
\hline & & 4 & 15 & 18 & 21 & 26 & 27 & 30 & 32 \\
\hline Pavona & decusssata & $\operatorname{TrC}$ & & & $\operatorname{TrV}$ & & & & \\
\hline Pavona & varians & $\operatorname{TrC}$ & & & & & & & \\
\hline Pectinia & paeonia & & & $\operatorname{TrC}$ & & & & & \\
\hline Platygyra & daedalea & & & $\operatorname{TrC}$ & & & $\operatorname{TrC}$ & & \\
\hline Platygyra & pini & $\operatorname{TrC}, \mathrm{C}$ & $\operatorname{TrC}$ & $\operatorname{TrC}$ & & & & & \\
\hline Pocillopora & damicomis & $\mathrm{TrV}$ & $\operatorname{TrC}$ & $\mathrm{TrV}$ & $\operatorname{TrV}$ & & & & \\
\hline Pocillopora & eydouxi & $\mathrm{C}$ & & & $\mathrm{C}$ & & & & \\
\hline Podabacia & crustacea & $\mathrm{C}$ & & & & & & & \\
\hline Polyphyllia & talpina & & & & & & $\operatorname{TrC}$ & & \\
\hline Porites & cylindrica & $\mathrm{C}$ & & $\operatorname{TrC}$ & & & & & \\
\hline Porites & heronensis & & & $\operatorname{TrC}$ & & & & & \\
\hline Porites & lichen & $\operatorname{TrC}$ & & & & & & & \\
\hline Porites & lobata & & & $\operatorname{TrC}$ & $\operatorname{Tr} C$ & $\operatorname{TrC}$ & & & \\
\hline Porites & lutea & $\operatorname{TrC}$ & & $\operatorname{TrC}$ & $\operatorname{TrC}$ & & $\operatorname{TrC}$ & & $\operatorname{TrV}$ \\
\hline Porites & rus & & & $\operatorname{TrC}$ & & & & & \\
\hline Stylocoeniella & guentheri & & & & $\operatorname{TrC}$ & & & & \\
\hline Symphyllia & recta & & & & & & $\operatorname{TrV}$ & & $\operatorname{TrV}$ \\
\hline Tubastraea & diaphana & & $\operatorname{TrC}, \mathrm{C}$ & & & $\operatorname{TrC}$ & & & \\
\hline Turbinaria & frondens & & & $\operatorname{TrC}$ & & & & & $\operatorname{TrV}$ \\
\hline Turbinaria & mesenterina & $\operatorname{TrC}, \mathrm{C}$ & & & & & & & \\
\hline Turbinaria & peltata & $\operatorname{TrC}, \mathrm{C}$ & & & & $\operatorname{TrC}$ & $\operatorname{TrC}$ & & $\operatorname{TrV}$ \\
\hline Turbinaria & reniformis & $\operatorname{TrC}$ & $\operatorname{TrC}$ & & & $\operatorname{TrV}$ & $\operatorname{TrC}$ & & $\operatorname{TrV}$ \\
\hline Turbinaria & stellulata & & & & & & $\operatorname{TrC}$ & & \\
\hline
\end{tabular}


Appendix 4 An updated list of zooxanthellate scleractinian corals at the Dampier Archipelago.*

* compiled from Hoeksema (1989), Simpson (1988), Veron (1993), Veron and Marsh (1988) and registration records of the Western Australian Museum. Names in bold italics indicate new records for the Dampier Archipelago collected during the present study.

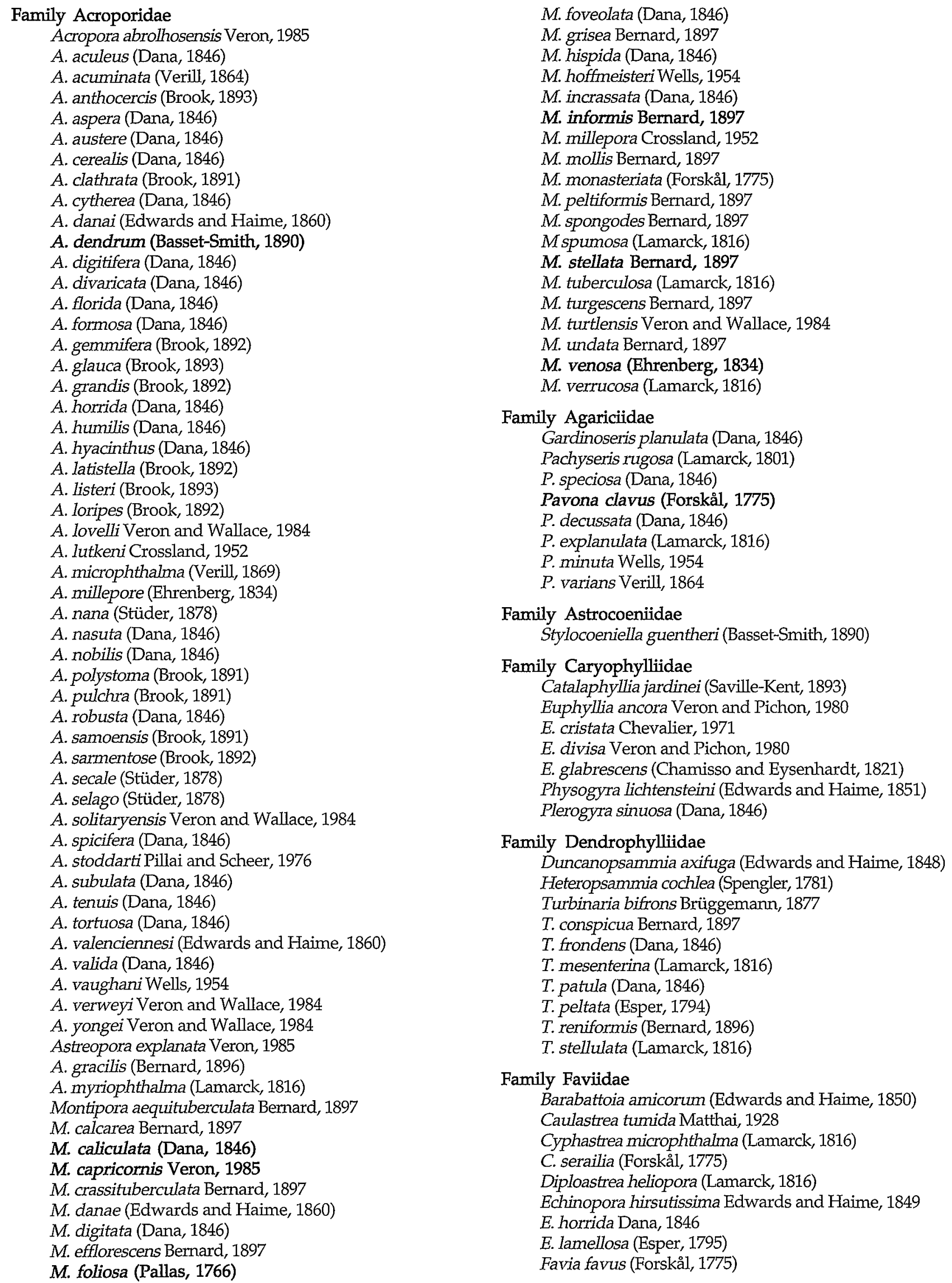


F. lizardensis Veron, Pichon and Wijsman-Best, 1977

F. matthaii Vaughan, 1918

F. maxima Veron, Pichon and Wijsman-Best, 1977

F. pallida (Dana, 1846)

F. rotumana (Gardiner, 1899)

F. rotundata Veron, Pichon and Wijsman-Best, 1977

F. speciosa (Dana, 1846)

F. stelligera (Dana, 1846)

F. veroni Moll and Borel Best, 1984

Favites abdita (Ellis and Solander, 1786)

F. chinensis (Verrill, 1866)

F. complanata (Ehrenberg, 1834)

F. flextiosa (Dana, 1846)

F. halicora (Ehrenberg, 1834)

F. pentagonna (Esper, 1794)

F. russelli (Wells, 1954)

Goniastrea aspera (Verrill, 1857)

G. australiensis Edwards and Haime, 1849

G. edwardsi Chevalier, 1971

G. favulus (Dana, 1846)

G. palauensis (Yabe, Sugiyama and Eguchi, 1936)

G. pectinata (Ehrenberg, 1834)

G. retiformis (Lamarck, 1816)

Hydnophora exesa (Pallas, 1766)

H. microconos (Lamarck, 1816)

H. pilosa Veron, 1985

H. rigida (Dana, 1846)

Leptastrea pruinosa Crossland, 1952

L. purpurea (Dana, 1846)

L. transversa Klunzinger, 1879

Leptoria phrygia (Ellis and Solander, 1786)

Montastrea curta (Dana, 1846)

M. magnistellata Chevalier, 1971

M. valenciennesi (Edwards and Haime, 1848)

Moseleya latistellata Quelch, 1884

Oulophyllia bennettae (Veron, Pichon and Wijsman Best, 1977)

O. crispa (Lamarck, 1816)

Platygyra daedalea (Ellis and Solander, 1786)

P. lamellina (Ehrenberg, 1834)

P. pini Chevalier, 1975

P. ryukyuensis (Yabe and Sugiyama, 1935)

P. sinensis (Edwards and Haime, 1849)

$P$. verveyi Wijsman-Best, 1976

Plesiastrea versipora (Lamarck, 1816)

\section{Family Fungiidae}

Fungia concinna Verrill, 1864

Fungia (Cycloseris) cyclolites (Lamarck, 1801)

F. fungites (Linnaeus, 1758)

F. repanda Dana, 1846

F. scutaria Lamarck, 1801

Herpolitha limax (Houttuyn, 1772)

Lithophyllon undulatum Rehberg, 1892

Podabacia crustacea (Pallas, 1766)

Polyphyllia talpina (Lamarck, 1801)

\section{Family Merulinidae}

Merulina ampliata (Ellis and Solander, 1786)

M. scabricula Dana, 1846

Scapophyllia cylindrica (Edwards and Haime, 1849)

\section{Family Mussidae}

Acanthastrea echinata (Dana, 1846)

A. hillae Wells, 1955

A. lordhowensis Veron and Pichon, 1982

Australomussa rowleyensis Veron, 1985
Blastomussa merleti (Wells, 1961)

Lobophyllia corymbosa (Forskål, 1775)

L. hataii Yabe, Sugiyama and Eguchi, 1936

L. hemprichii (Ehrenberg, 1834)

Symphyllia agaricia (Edwards and Haime, 1848)

S. recta (Dana, 1846)

S. valenciennesii Edwards and Haime, 1849

Family Oculinidae

Galaxea astreata (Lamarck, 1816)

G. fascicularis (Linnaeus, 1758)

Family Pectiniidae

Echinophyllia aspera (Ellis and Solander, 1786)

E. orpheensis Veron and Pichon, 1980

Mycedium elephantotus (Pallas, 1766)

Oxypora lacera (Verrill, 1864)

Pectinia lactuca (Pallas, 1766)

P. paeonia (Dana, 1846)

Family Pocilloporidae

Pocillopora damicornis (Linnaeus, 1758)

P. eydouxi Edwards and Haime, 1860

P. meandrina Dana, 1846

$P$. verrucosa (Ellis and Solander, 1786)

P. woodjonesi Vaughan, 1918

Seriatopora caliendrum (Ehrenberg, 1834)

Stylophora pistillata (Esper, 1794)

Family Poritidae

Alveopora fenestrata (Lamarck, 1816)

Goniopora columna Dana, 1846

G. djiboutensis Vaughan, 1907

G. lobata Edwards and Haime, 1860

G. minor Crossland, 1952

G. palmensis Veron and Pichon, 1982

G. pandoraensis Veron and Pichon, 1982

G. pendulus Veron, 1985

G. stokesi Edwards and Haime, 1860

G. stutchburyi Wells, 1955

G. tenuidens Quelch, 1886

Porites aranetai Nemenzo, 1955

P. cylindrica Dana, 1846

P. evermanni Vaughan, 1907

P. heronensis Veron, 1985

P. lichen Dana, 1846

P. lobata Dana, 1846

P. Iutea Edwards and Haime, 1860

P. murrayensis Vaughan, 1918

P. rus (Forskål, 1775)

P. solida (Forskål, 1775)

Family Siderastreidae

Coscinaraea columna (Dana, 1846)

C. exesa (Dana, 1846)

Pseudosiderastrea tayamai Yabe and Sugiyama, 1935

Family Thamnasteriidae

Psammocora contigua (Esper, 1797)

P. digitata Edwards and Haime, 1851

P. explanulata van der Horst, 1922

P. haimeana Edwards and Haime, 1851

P. nierstraszi van der Horst, 1921

P. profundacella Gardiner, 1898

P. superficialis Gardiner, 1898

Family Trachyphylliidae

Trachyphyllia geoffroyi (Audouin, 1826) 
Appendix 5 A list of azooxanthellate scleractinians from the Dampier Archipelago.* * compiled from Cairns (1998) and registration records of the Western Australian Museum.

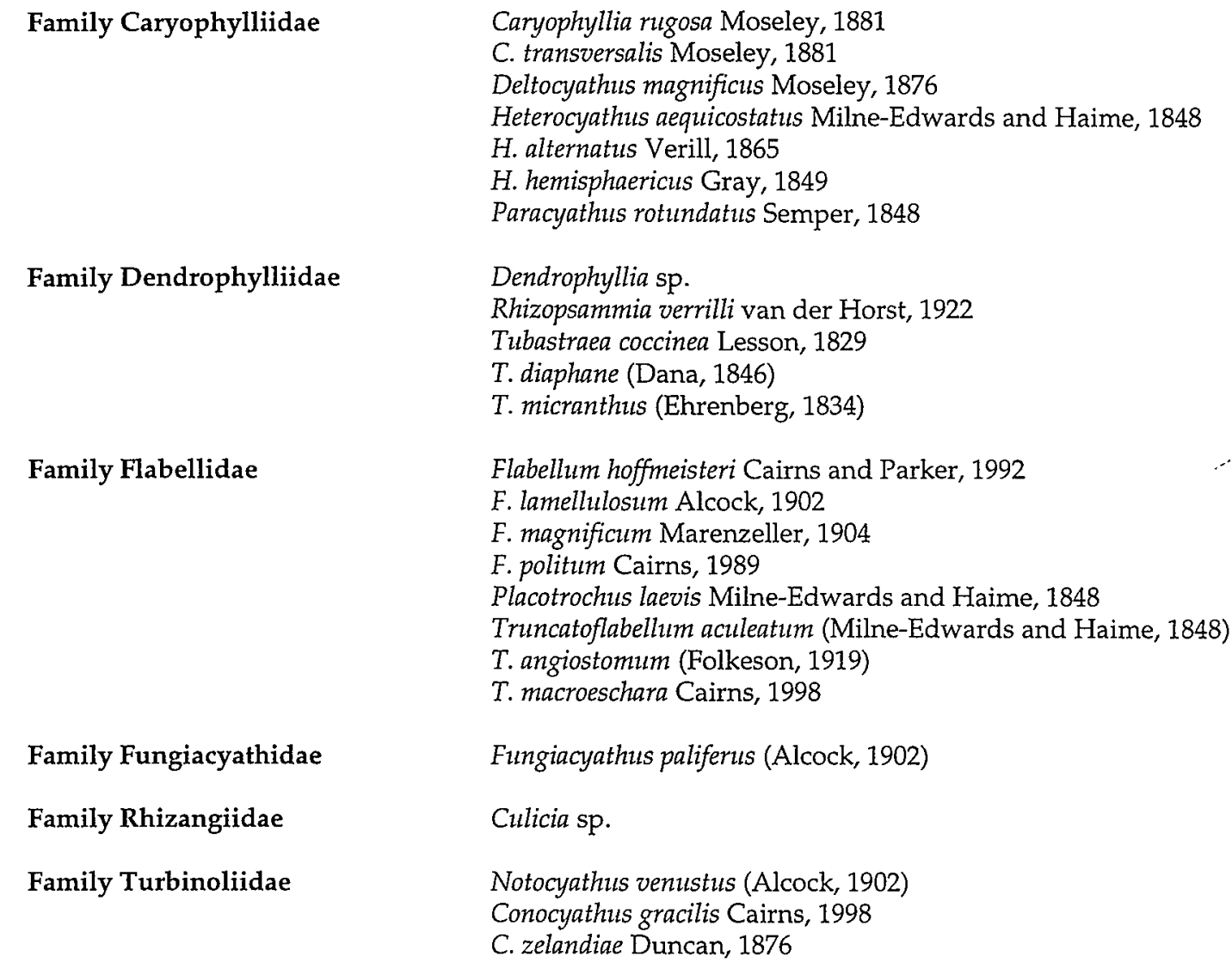

Family Flabellidae

Family Dendrophylliidae

Dendrophyllia sp.

Rhizopsammia verrilli van der Horst, 1922

Tubastraea coccinea Lesson, 1829

T. diaphane (Dana, 1846)

T. micranthus (Ehrenberg, 1834)

Flabellum hoffmeisteri Cairns and Parker, 1992

F. lamellulosum Alcock, 1902

F. magnificum Marenzeller, 1904

F. politum Cairns, 1989

Placotrochus laevis Milne-Edwards and Haime, 1848

Truncatoflabellum aculeatum (Milne-Edwards and Haime, 1848)

T. angiostomum (Folkeson, 1919)

T. macroeschara Cairns, 1998

Fungiacyathus paliferus (Alcock, 1902)

Culicia sp.

Notocyathus venustus (Alcock, 1902)

Conocyathus gracilis Cairns, 1998

C. zelandiae Duncan, 1876 